Systematic Review

\title{
Systematic Review of the Effectiveness of Thermal Annular Procedures in Treating Discogenic Low Back Pain
}

Standiford Helm II, MD¹, Salim M. Hayek, MD, PhD², Ramsin Benyamin, MD², and Laxmaiah Manchikanti, MD ${ }^{4}$

From: ${ }^{1}$ Pacific Coast Pain Management Center, Laguna Hills, CA; ${ }^{2}$ University Hospitals of Cleveland and Outcomes Research Consortium, Cleveland, $\mathrm{OH}$; ${ }^{3}$ Millennium Pain Center, Bloomington, IL; and 4Pain Management Center of Paducah, Paducah, KY.

Dr. Helm is a Medical Director, Pacific Coast Pain Management Center, Laguna Hills, CA. Dr. Hayek is Chief of the Division of Pain Medicine, Department of Anesthesiology, University Hospitals of Cleveland,

Cleveland, $\mathrm{OH}$; and a member of the Outcomes Research Consortium, Cleveland, $\mathrm{OH}$.

Dr. Benyamin is President, Millennium Pain Center, Clinical Associate Professor, Department of Surgery, College of Medicine, University of Illinois, Urbana-Champaign, IL.

Dr. Manchikanti is Medical Director of the Pain

Management Center of Paducah,

$\mathrm{KY}$, and Associate Clinical Professor of Anesthesiology and Perioperative Medicine, University of Louisville, Louisville, KY.

Address correspondence: Standiford Helm II, MD 24902 Moulton Pkwy, Suite 200 Laguna Hills, CA 92637

E-mail: drhelm@pcpmc.com

Disclaimer: There was no external funding in the preparation of this manuscript. Conflict of interest: None.

Manuscript received: $10 / 1 / 2008$ Accepted for publication: $11 / 22 / 2008$

Free full manuscript: www.painphysicianjournal.com
Background: Chronic discogenic low back pain is a common problem with significant personal and societal costs. Thermal annular procedures (TAPs) have been developed in an effort to provide a minimally invasive treatment for this disorder. Multiple techniques utilized are intradiscal electrothermal therapy (IDET), radiofrequency annuloplasty, and intradiscal biacuplasty (IDB). However, these treatments continue to be controversial, coupled with a paucity of evidence.

Study Design: A systematic review of the literature evaluating the efficacy or effectiveness of TAPs.

Objective: To determine the effectiveness of TAPs in reducing low back pain in patients with intradiscal disorders.

Methods: A comprehensive evaluation of the literature relating to TAPs was performed. The literature was evaluated according to Cochrane Review criteria for randomized controlled trials (RCTs) and according to the Agency for Healthcare Research and Quality (AHRQ) criteria for observational studies

The level of evidence was classified as Level I, II, or III based on the quality of evidence developed by the U.S. Preventive Services Task Force (USPSTF). Pain relief was the primary outcome measure. Other outcome measures were functional improvement, improvement of psychological status, and return to work.

Data sources included relevant literature of the English language identified through searches of PubMed, EMBASE, the Cochrane Library, and the Database of Reviews of Effectiveness (DARE)

Outcome Measures: Short-term effectiveness was defined as one-year or less and long-term effectiveness was defined as greater than one-year.

Results: Systematic review of IDET identified 2 RCTs and 16 observational studies with an indicated evidence of Level II-2.

Systematic review of radiofrequency annuloplasty identified no RCTs but 2 observational studies with an uncertain evidence of Level II-3.

Systematic review of IDB identified one pilot study. The level of evidence is lacking with Level III.

Limitations: The limitations of this review include paucity of the literature and lack of evidence with internal validity and generalizability.

Conclusion: IDET offers functionally significant relief in approximately one-half of appropriately chosen chronic discogenic low back pain patients. There is minimal evidence supporting the use of radiofrequency annuloplasty and IDB.

Key words: Chronic low back pain, degenerative disc disease, internal disc disruption, intervertebral disc, thermal annular procedures, intradiscal electrothermal therapy, radiofrequency ablation, intradiscal biacuplasty, radiofrequency annuloplasty

Pain Physician 2009; 12:1:207-232 


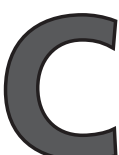
hronic low back pain is a common problem with a prevalence ranging from $35 \%$ to $75 \%$ at 12 months after the initial attack of pain $(1,2)$. The widely held myth that $90 \%$ of low back pain is short-lived and that most patients get better on their own has been dispelled in multiple studies (3-7). The anatomical sources of persistent low back pain have been defined and include the lumbar intervertebral discs, facet or zygapophysial joints, and the sacroiliac joint (8-12). Disorders of the intervertebral disc have been estimated to be the cause of persistent low back pain in $7 \%, 39 \%$, and $26 \%$ of patients in various studies (9-11). Valuable information pertaining to the diagnosis of discogenic low back pain could be achieved by performing provocation discography (12). However, treatment of discogenic low back pain can be frustrating, with neither conservative treatment nor fusion reliably resolving the problem $(13,14)$.

To provide an alternative between failed conservative therapy and fusion, several technologies have been developed to apply heat to the posterior lumbar annulus. The rationale is that the degenerated disc has the ability to generate pain and that the application of heat can resolve this pain. The lumbar intervertebral disc consists of a central nucleus pulposus, a surrounding annulus fibrosis, and the endplates. The annulus is composed of ordered collagen fibers $(15,16)$. With aging, the disc undergoes degeneration in the form of delamination of the annular layers like tearing, along with endplate changes which reduce diffusion. These changes are age related and have the ability to generate pain (17-19).

This degeneration is ubiquitous and begins to occur as early as age 11 (20). Degeneration appears to cause pain because of the development of granulation tissue and nerve endings in the fissures (21). The normal annulus and posterior longitudinal ligament are innervated and nerve growth in normal discs is limited to the superficial $3.5 \mathrm{~mm}$ of the disc (22-26). Degenerated discs have increased innervation compared to normal discs and are a source of back pain $(27,28)$. Substance $P$ is found in degenerated discs, providing an anatomic basis for pain perception from the disc $(29,30)$. Mechanoreceptors are also present. Nerve supply from either the sinuvertebral nerves or the paravertebral sympathetic trunks entering the dorsal root ganglia at L2 or above has been demonstrated (27,31-35). Aoki et al (36) found that gene-related peptide-immunoreceptive neurons proliferated in inflamed discs, suggesting these neurons were responsible for pain from intradiscal disorders. These receptors appear to be sensitized by the production of proinflammatory cytokines and mediators such as prostaglandin E2, and interleukin (IL)-6 and (IL)-8, so that pressure directly stimulates the nociceptors $(21,37)$. Painful discs also show evidence of injury and repair not found in aged, non-painful discs (38).

The current hypothesis, therefore, is that the inflammatory response associated with the onset of annular fissures sensitizes the nerves that innervate the fissures, leading to pain. Intradiscally applied heat has been used to treat this pain. Despite the ongoing clarification as to the mechanisms by which intradiscal pain occurs, the mechanisms by which heat relieves pain emanating from the disc are unclear. Derby et al (39) have reviewed the proposed mechanisms of action, including changes in disc biomechanics, annular contraction, thermally induced healing response, sealing of annular tears, annular denervation, and decreased intradiscal disorder, with the conclusion that the mechanism of pain relief is unclear. Derby et al (39) hypothesize that if patients with no or a minimal (< 1 week) flare of pain after the procedure do better, then that finding would be consistent with a denervation mechanism. Kapural et al (40) have shown that intradiscal biacuplasty (IDB) generates sufficient annular temperatures for neuroablation, suggesting that the pain relief is caused by denervation.

The first technology to apply heat was IDET, using convection heating, first applied in 1996 (41). IDET utilizes a $5 \mathrm{~cm}$ active tip catheter placed in the nuclearannular junction or in the posterior annulus. Finch et al (42) developed a technique using monopolar radiofrequency energy to apply ionic heating to the posterior annulus. Recently, bipolar cooled radiofrequency, with electrodes placed on both sides, has been used to treat the posterior annulus (40).

Percutaneous intradiscal treatment of low back pain has been the subject of several reviews (4350). The Centers for Medicare and Medicaid Services (CMS) has recently issued a non-certification for these procedures (50). CMS refers to them collectively as thermal intradiscal procedures, including intradiscal electrothermal therapy (IDET), percutaneous intradiscal radiofrequency thermocoagulation (PIRFT), radiofrequency annuloplasty, intradiscal biacuplasty (IDB), percutaneous (or plasma) disc decompression (PDD) or coblation, or targeted disc decompression (TDD).

This systematic review is undertaken to evaluate the current evidence of thermal annular procedures (TAPs). The current review focuses on heat treatment 
of the annulus. Accordingly, only IDET, radiofrequency annuloplasty, and IDB are covered here. Since we are referring to heat treatment of the annulus rather than any heat treatment within the disc, these procedures are collectively referred to as TAPs.

\section{Methods}

\section{Literature Search}

Databases reviewed were PubMed, EMBASE, the Cochrane Library, and the Database of Reviews of Effectiveness (DARE). Bibliographies of reviewed papers were also examined. In addition, authors known to be active in the field were contacted. The time frame covered was 1966 to November 2008.

Inclusion criteria were:

1. Lumbar intradiscal pain of at least 6 months duration;

2. Treatment with an annuloplasty procedure using IDET, radiofrequency annuloplasty, or IDB;

3. Minimum of 6 month follow-up.
Search terms included intervertebral disc, degenerative disc disease, intradiscal electrothermal therapy (IDET), radiofrequency ablation, annuloplasty, internal disc disruption, and thermal intradiscal procedures.

Only articles in English or with English abstracts, systematic reviews, randomized controlled trials (RCTs), and observational studies were reviewed. All data extraction was performed by one author (SH). Each article was reviewed by 2 reviewers. Discrepancies in rating were resolved by adjudication by a third reviewer. If there was a conflict of interest with the reviewed manuscripts such as authorship or any other type of conflict, the involved authors did not review the manuscripts for quality assessment, clinical relevance, evidence synthesis, or grading of evidence.

\section{Methodologic Quality Assessment}

The method of quality assessment was a function of the type of study. For RCTs, the Cochrane review criteria were used (Table 1) (51). Assessment of study quality for observational studies was done according

Table 1. Modified and weighted Cochrane methodologic quality assessment criteria.

\begin{tabular}{|c|c|c|}
\hline \multicolumn{2}{|r|}{ CRITERION } & Weighted Score (points) \\
\hline \multicolumn{2}{|c|}{ 1. Study population } & 35 \\
\hline A & Homogeneity & 2 \\
\hline B & Comparability of relevant baseline characteristics & 5 \\
\hline $\mathrm{C}$ & Randomization procedure adequate & 4 \\
\hline $\mathrm{D}$ & Drop-outs described for each study group separately & 3 \\
\hline \multirow[t]{2}{*}{ E } & $<20 \%$ loss for follow-up & 2 \\
\hline & $<10 \%$ loss for follow-up & 2 \\
\hline \multirow[t]{2}{*}{$\mathrm{F}$} & $>50$ subject in the smallest group & 8 \\
\hline & $>100$ subjects in the smallest group & 9 \\
\hline \multicolumn{2}{|c|}{ 2. Interventions } & 25 \\
\hline G & Interventions included in protocol and described & 10 \\
\hline $\mathrm{H}$ & Pragmatic study & 5 \\
\hline I & Co-interventions avoided or similar & 5 \\
\hline $\mathrm{J}$ & Placebo-controlled & 5 \\
\hline \multicolumn{2}{|c|}{ 3. Effect } & 30 \\
\hline $\mathrm{K}$ & Patients blinded & 5 \\
\hline $\mathrm{L}$ & Outcome measures relevant & 10 \\
\hline M & Blinded outcome assessments & 10 \\
\hline $\mathrm{N}$ & Follow-up period adequate & 5 \\
\hline \multicolumn{2}{|c|}{ 4. Data-presentation and analysis } & 10 \\
\hline $\mathrm{O}$ & Intention-to-treat analysis & 5 \\
\hline $\mathrm{P}$ & Frequencies of most important outcomes presented for each treatment group & 5 \\
\hline \multicolumn{2}{|r|}{$\begin{array}{|lc|} & \text { TOTAL SCORE } \\
\end{array}$} & 100 \\
\hline
\end{tabular}

Adapted from Koes BW et al. Efficacy of epidural steroid injections for low-back pain and sciatica: A systematic review of randomized clinical trials. Pain 1995; 63:279-288 (51). 
to the Agency for Healthcare Research and Quality (AHRQ) criteria (Table 2) (52). Both the RCTs and obser- vational forms provide a maximum of 100 points; only studies with scores of over 50 points were included.

Table 2. Modified AHRQ quality assessment criteria for observational studies.

\begin{tabular}{|c|c|}
\hline CRITERION & Weighted Score (points) \\
\hline 1. Study Question & 2 \\
\hline \multicolumn{2}{|l|}{ - Clearly focused and appropriate question } \\
\hline 2. Study Population & 8 \\
\hline - Description of study population & 5 \\
\hline - Sample size justification & 3 \\
\hline 3. Comparability of Subjects & 22 \\
\hline - Specific inclusion/exclusion criteria for all groups & 5 \\
\hline - Criteria applied equally to all groups & 3 \\
\hline - Comparability of groups at baseline with regard to disease status and prognostic factors & 3 \\
\hline - Study groups comparable to non-participants with regard to confounding factors & 3 \\
\hline - Use of concurrent controls & 5 \\
\hline - Comparability of follow-up among groups at each assessment & 3 \\
\hline 4. Exposure or Intervention & 11 \\
\hline - Clear definition of exposure & 5 \\
\hline - Measurement method standard, valid and reliable & 3 \\
\hline - Exposure measured equally in all study groups & 3 \\
\hline 5. Outcome measures & 20 \\
\hline - Primary/secondary outcomes clearly defined & 5 \\
\hline - Outcomes assessed blind to exposure or intervention & 5 \\
\hline - Method of outcome assessment standard, valid and reliable & 5 \\
\hline - Length of follow-up adequate for question & 5 \\
\hline 6. Statistical Analysis & 19 \\
\hline - Statistical tests appropriate & 5 \\
\hline - Multiple comparisons taken into consideration & 3 \\
\hline - Modeling and multivariate techniques appropriate & 2 \\
\hline - Power calculation provided & 2 \\
\hline - Assessment of confounding & 5 \\
\hline - Dose-response assessment if appropriate & 2 \\
\hline 7. Results & 8 \\
\hline - Measure of effect for outcomes and appropriate measure of precision & 5 \\
\hline - Adequacy of follow-up for each study group & 3 \\
\hline 8. Discussion & 5 \\
\hline \multicolumn{2}{|l|}{ - Conclusions supported by results with possible biases and limitations taken into consideration } \\
\hline 9. Funding or Sponsorship & 5 \\
\hline \multicolumn{2}{|l|}{ - Type and sources of support for study } \\
\hline TOTAL SCORE & 100 \\
\hline
\end{tabular}

Adapted and modified from West S et al. Systems to Rate the Strength of Scientific Evidence, Evidence Report, Technology Assessment No. 47. AHRQ Publication No. 02-E016 (52). 
Consensus-based weighted scoring developed by the Guidelines Committee of the American Society of Interventional Pain Physicians (ASIPP) was utilized. The same scoring system has been used in multiple evaluations $(49,53-57)$.

\section{Clinical Relevance}

Clinical relevance of the included studies was evaluated according to 5 questions recommended by the Cochrane Back Review Group $(58,59)$.

Table 3 shows the clinical relevance questions. Each question was scored positive (+) if the clinical relevance item was met, negative $(-)$ if the item was not met, and unclear (?) if data were not available to answer the question.

In the Cochrane review of "Injection Therapy for Subacute and Chronic Low Back Pain" (59) the authors considered a $20 \%$ improvement in pain scores (60) and a $10 \%$ improvement in functioning outcomes (61) to be clinically important.

Both RCTs and observational studies were included in the review to improve generalizability and application of the TAPs (62-68).

\section{Outcome Measures}

Pain relief was the primary outcome measure. Other outcome measures were functional improvement, improvement of psychological status, and return to work.

A decrease of either 2 points or $30 \%$ of pain scores provides a useful benchmark of clinical importance to assess effectiveness $(60,69)$. Similarly, a $10 \%$ improvement in functioning outcomes provides an accepted benchmark of clinically useful benefit (61). However, in interventional pain management settings, a significant improvement has been defined as $50 \%$ or more relief, whereas significant improvement in disability has been defined as a $40 \%$ or more decrease in disability scores in multiple publications $(62-68,70-76)$.

\section{Analysis of Evidence}

Level of evidence was determined based on the United States Preventive Services Task Force (USPSTF) criteria using 5 levels of evidence, ranging from Level I to III with 3 subcategories in Level II, as illustrated in Table 4 (77).

Table 3. Clinical relevance questions.

\begin{tabular}{|l||}
\hline A) Are the patients described in detail so that you can decide whether they are comparable to those that you see in your practice? \\
\hline B) Are the interventions and treatment settings described well enough so that you can provide the same for your patients? \\
\hline C) Were all clinically relevant outcomes measured and reported? \\
\hline D) Is the size of the effect clinically important? \\
\hline E) Are the likely treatment benefits worth the potential harms? \\
\hline
\end{tabular}

Source: Staal JB et al. Injection therapy for subacute and chronic low-back pain. Cochrane Database Syst Rev 2008; 3:CD001824 (59).

Table 4. Quality of evidence developed by USPSTF.

\begin{tabular}{|c|l||}
\hline I: & Evidence obtained from at least one properly randomized controlled trial \\
\hline II-1: & Evidence obtained from well-designed controlled trials without randomization \\
\hline II-2: & $\begin{array}{l}\text { Evidence obtained from well-designed cohort or case-control analytic studies, preferably from more than one } \\
\text { center or research group }\end{array}$ \\
\hline II-3: & $\begin{array}{l}\text { Evidence obtained from multiple time series with or without the intervention. Dramatic results in uncontrolled } \\
\text { experiments (such as the results of the introduction of penicillin treatment in the 1940s) could also be regarded } \\
\text { as this type of evidence }\end{array}$ \\
\hline III: & $\begin{array}{l}\text { Opinions of respected authorities, based on clinical experience descriptive studies and case reports or reports of } \\
\text { expert committees }\end{array}$ \\
\hline
\end{tabular}

Adapted from the U.S. Preventive Services Task Force (USPSTF) (77). 


\section{Recommendations}

Recommendations for effectiveness were made according to Guyatt et al's criteria (78) (Table 5).

Data will be analyzed for both short-term (1 year or less) and long-term (longer than 1 year).

\section{Results}

The results of literature search for thermal annular procedures (TAPs) are illustrated in Fig. 1.

A total of 67 articles were located in the literature search. Of these, 36 were RCTs or observational studies.

\section{Methodologic Quality Assessment}

\section{Randomized Controlled Trials}

Of the RCTs, 2 studies met inclusion criteria with methodologic quality assessment scores illustrated in Table 6. The scores were 61 of 100 for Freeman et al (79) and 68 of 100 for Pauza et al (80).

\section{Clinical Relevance Assessment}

Both studies $(79,80)$ met clinical relevance criteria as shown in Table 7. Both studies have been criticized $(81,82)$. Despite these criticisms, both describe patients in sufficient detail for a practitioner to identify them in a clinical setting. Both describe IDET sufficiently that the procedure can be provided outside of the academic setting. Both measured and reported clinically relevant effects. Pauza et al (80) did meet all the criteria for clinically important improvement, including a greater than $30 \%$ improvement in pain scores, a 2-point reduction in visual analog score (VAS) in about $50 \%$ of patients, and a greater than $10 \%$ improvement in functioning scores, although the functioning score improvement was not clinically significant. According to Pauza et al (80), but not according to Freeman et al (79), the benefits of TAP are worth the potential harms.

\section{Observational Studies}

Overall 34 observational studies met the inclusion criteria for methodologic quality assessment (41,42,83-115). However, of these, methodologic quality assessment was performed on 31 studies after combining duplicate studies. Methodologic quality scores are described in Table 8, ranging from 35 to 85 . Of these, 20 studies scored 50 or above $(41,42,83-92,94-101,103,114,115)$, meeting

Table 5. Grading recommendations.

\begin{tabular}{|c|c|c|c|}
\hline $\begin{array}{l}\text { Grade of } \\
\text { Recommendation/ } \\
\text { Description }\end{array}$ & $\begin{array}{l}\text { Benefit vs Risk and } \\
\text { Burdens }\end{array}$ & $\begin{array}{l}\text { Methodological Quality of } \\
\text { Supporting Evidence }\end{array}$ & Implications \\
\hline $\begin{array}{l}\text { 1A/strong recommendation, } \\
\text { high-quality evidence }\end{array}$ & $\begin{array}{l}\text { Benefits clearly outweigh risk } \\
\text { and burdens, or vice versa }\end{array}$ & $\begin{array}{l}\text { RCTs without important limitations or } \\
\text { overwhelming evidence from observa- } \\
\text { tional studies }\end{array}$ & $\begin{array}{l}\text { Strong recommendation, can ap- } \\
\text { ply to most patients in most cir- } \\
\text { cumstances without reservation }\end{array}$ \\
\hline $\begin{array}{l}\text { 1B/strong recommendation, } \\
\text { moderate quality evidence }\end{array}$ & $\begin{array}{l}\text { Benefits clearly outweigh risk } \\
\text { and burdens, or vice versa }\end{array}$ & $\begin{array}{l}\text { RCTs with important limitations (incon- } \\
\text { sistent results, methodological flaws, indi- } \\
\text { rect, or imprecise) or exceptionally strong } \\
\text { evidence from observational studies }\end{array}$ & $\begin{array}{l}\text { Strong recommendation, can ap- } \\
\text { ply to most patients in most cir- } \\
\text { cumstances without reservation }\end{array}$ \\
\hline $\begin{array}{l}1 \mathrm{C} / \text { strong recommendation, } \\
\text { low-quality or very low-qual- } \\
\text { ity evidence }\end{array}$ & $\begin{array}{l}\text { Benefits clearly outweigh risk } \\
\text { and burdens, or vice versa }\end{array}$ & Observational studies or case series & $\begin{array}{l}\text { Strong recommendation but } \\
\text { may change when higher qual- } \\
\text { ity evidence becomes available }\end{array}$ \\
\hline $\begin{array}{l}\text { 2A/weak recommendation, } \\
\text { high-quality evidence }\end{array}$ & $\begin{array}{l}\text { Benefits closely balanced } \\
\text { with risks and burden }\end{array}$ & $\begin{array}{l}\text { RCTs without important limitations or } \\
\text { overwhelming evidence from observa- } \\
\text { tional studies }\end{array}$ & $\begin{array}{l}\text { Weak recommendation, best action } \\
\text { may differ depending on circum- } \\
\text { stances or patients' or societal values }\end{array}$ \\
\hline $\begin{array}{l}\text { 2B/weak recommendation, } \\
\text { moderate-quality evidence }\end{array}$ & $\begin{array}{l}\text { Benefits closely balanced } \\
\text { with risks and burden }\end{array}$ & $\begin{array}{l}\text { RCTs with important limitations (incon- } \\
\text { sistent results, methodological flaws, indi- } \\
\text { rect, or imprecise) or exceptionally strong } \\
\text { evidence from observational studies }\end{array}$ & $\begin{array}{l}\text { Weak recommendation, best } \\
\text { action may differ depending on } \\
\text { circumstances or patients' or } \\
\text { societal values }\end{array}$ \\
\hline $\begin{array}{l}2 \mathrm{C} / \text { weak recommendation, } \\
\text { low-quality or very low-qual- } \\
\text { ity evidence }\end{array}$ & $\begin{array}{l}\text { Uncertainty in the estimates } \\
\text { of benefits, risks, and burden; } \\
\text { benefits, risk, and burden may } \\
\text { be closely balanced }\end{array}$ & Observational studies or case series & $\begin{array}{l}\text { Very weak recommendations; } \\
\text { other alternatives may be } \\
\text { equally reasonable }\end{array}$ \\
\hline
\end{tabular}

Adapted from Guyatt $\mathrm{G}$ et al. Grading strength of recommendations and quality of evidence in clinical guidelines. Report from an American College of Chest Physicians task force. Chest 2006; 129:174-181 (78). 


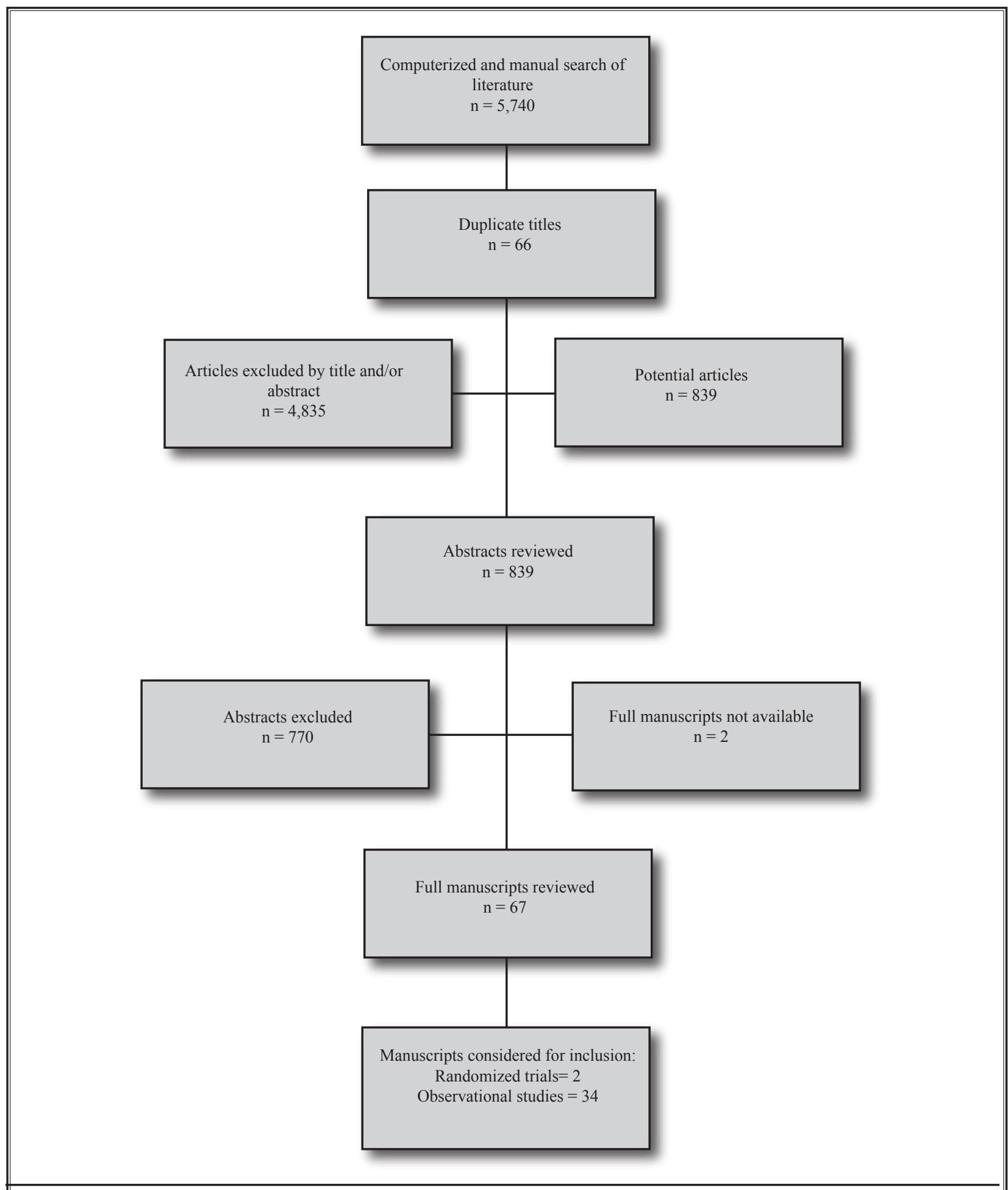

Fig. 1. The flow diagram illustrating literature evaluating thermal annular procedures (TAPs). 
Table 6. Methodological assessment of randomized clinical trials evaluating the effectiveness of TAPS.

\begin{tabular}{|c|c|c|c|c|}
\hline \multicolumn{2}{|r|}{ CRITERION } & $\begin{array}{l}\text { Weighted } \\
\text { Score }\end{array}$ & $\begin{array}{l}\text { Pauza et al } \\
\quad(\mathbf{8 0})\end{array}$ & $\begin{array}{c}\text { Freeman et al } \\
(79)\end{array}$ \\
\hline \multicolumn{5}{|c|}{ Study population } \\
\hline A & Homogeneity & 2 & 2 & 0 \\
\hline $\mathrm{B}$ & Comparability of relevant baseline characteristics & 5 & 5 & 0 \\
\hline $\mathrm{C}$ & Randomization procedure adequate & 4 & 4 & 2 \\
\hline $\mathrm{D}$ & Drop-outs described for each study group separately & 3 & 3 & 3 \\
\hline \multirow[t]{2}{*}{$\mathrm{E}$} & $<20 \%$ loss for follow-up & 2 & 2 & 2 \\
\hline & $<10 \%$ loss for follow-up & 2 & 0 & 2 \\
\hline \multirow[t]{2}{*}{$\mathrm{F}$} & $>50$ subject in the smallest group & 8 & 0 & 0 \\
\hline & $>100$ subjects in the smallest group & 9 & 0 & 0 \\
\hline \multicolumn{5}{|c|}{ Interventions } \\
\hline G & Interventions included in protocol and described & 10 & 10 & 10 \\
\hline $\mathrm{H}$ & Pragmatic study & 5 & 0 & 5 \\
\hline I & Co-interventions avoided or similar & 5 & 5 & 5 \\
\hline $\mathrm{J}$ & Placebo-controlled & 5 & 5 & 0 \\
\hline \multicolumn{5}{|c|}{ Effect } \\
\hline K & Patients blinded & 5 & 5 & 5 \\
\hline $\mathrm{L}$ & Outcome measures relevant & 10 & 10 & 10 \\
\hline M & Blinded outcome assessments & 10 & 10 & 10 \\
\hline $\mathrm{N}$ & Follow-up period adequate & 5 & 2 & 2 \\
\hline \multicolumn{5}{|c|}{ Data-presentation and analysis } \\
\hline $\mathrm{O}$ & Intention-to-treat analysis & 5 & 0 & 0 \\
\hline $\mathrm{P}$ & $\begin{array}{l}\text { Frequencies of most important outcomes presented for } \\
\text { each treatment group }\end{array}$ & 5 & 5 & 5 \\
\hline & TOTAL SCORE & 100 & 68 & 61 \\
\hline
\end{tabular}

Table 7. Clinical relevance of randomized clinical trials evaluating the effectiveness of IDET.

\begin{tabular}{|l|c|c|}
\hline & Freeman et al (79) & Pauza et al (80) \\
\hline $\begin{array}{l}\text { A) Are the patients described in detail so that you can decide whether they are comparable to } \\
\text { those that you see in your practice? }\end{array}$ & + & + \\
\hline $\begin{array}{l}\text { B) Are the interventions and treatment settings described well enough so that you can provide } \\
\text { the same for your patients? }\end{array}$ & + & + \\
\hline C) Were all clinically relevant outcomes measured and reported? & - & + \\
\hline D) Is the size of the effect clinically important? & - & - \\
\hline E) Are the likely treatment benefits worth the potential harms? & $3 / 5$ & + \\
\hline TOTAL CRITERIA MET & + & $4 / 5$ \\
\hline
\end{tabular}

$+=$ positive; - = negative

Scoring adapted from Staal JB et al. Injection therapy for subacute and chronic low-back pain. Cochrane Database Syst Rev 2008; 3:CD001824 (59). 
Thermal Annular Procedures

Table 8. Methodological assessment of observational studies evaluating the effectiveness of TAPS.

\begin{tabular}{|c|c|c|c|c|c|c|c|c|c|c|}
\hline CRITERION & $\begin{array}{l}\text { Weight- } \\
\text { ed Score } \\
\text { (points) }\end{array}$ & $\begin{array}{l}\text { Assietti } \\
(105)\end{array}$ & $\begin{array}{l}\text { Bogduk } \\
\text { and } \\
\text { Karasek } \\
(90,115)\end{array}$ & $\begin{array}{l}\text { Bryce } \\
\text { et al } \\
(84)\end{array}$ & $\begin{array}{l}\text { Cohen } \\
\text { et al } \\
(85)\end{array}$ & $\begin{array}{l}\text { Davis } \\
\text { et al } \\
(114)\end{array}$ & $\begin{array}{c}\text { Derby } \\
\text { et al } \\
(102)\end{array}$ & $\begin{array}{l}\text { Derby } \\
\text { et al } \\
(86)\end{array}$ & $\begin{array}{l}\text { Derby } \\
\text { et al } \\
(87)\end{array}$ & $\begin{array}{l}\text { Derby } \\
\text { et al } \\
(109)\end{array}$ \\
\hline 1. Study Question & 2 & 2 & 2 & 2 & 2 & 2 & 2 & 2 & 2 & 2 \\
\hline - Clearly focused and appropriate question & & 2 & 2 & 2 & 2 & 2 & 2 & 2 & 2 & 2 \\
\hline 2. Study Population & 8 & 5 & 5 & 5 & 5 & 4 & 5 & 4 & 5 & 5 \\
\hline - Description of study population & 5 & 5 & 5 & 5 & 5 & 4 & 5 & 4 & 5 & 5 \\
\hline - Sample size justification & 3 & 0 & 0 & 0 & 0 & 0 & 0 & 0 & 0 & 0 \\
\hline $\begin{array}{l}\text { 3. Comparability of Subjects for All Observational } \\
\text { Studies }\end{array}$ & 22 & 3 & 19 & 5 & 11 & 5 & 5 & 13 & 5 & 5 \\
\hline - Specific inclusion/exclusion criteria for all groups & 5 & 3 & 5 & 5 & 5 & 0 & 5 & 5 & 5 & 5 \\
\hline - Criteria applied equally to all groups & 3 & 0 & 3 & 0 & 3 & 0 & 0 & 2 & 0 & 0 \\
\hline $\begin{array}{l}\text { - Comparability of groups at baseline with regard to } \\
\text { disease status and prognostic factors }\end{array}$ & 3 & 0 & 3 & 0 & 0 & 0 & 0 & 3 & 0 & 0 \\
\hline $\begin{array}{l}\text { - Study groups comparable to non-participants with } \\
\text { regard to confounding factors }\end{array}$ & 3 & 0 & 3 & 0 & 0 & 0 & 0 & 0 & 0 & 0 \\
\hline - Use of concurrent controls & 5 & 0 & 3 & 0 & 0 & 0 & 0 & 0 & 0 & 0 \\
\hline $\begin{array}{l}\text { - Comparability of follow-up among groups at each } \\
\text { assessment }\end{array}$ & 3 & 0 & 2 & 0 & 3 & 0 & 0 & 3 & 0 & 0 \\
\hline 4. Exposure or Intervention & 11 & 8 & 11 & 8 & 8 & 8 & 8 & 11 & 8 & 8 \\
\hline - Clear definition of exposure & 5 & 5 & 5 & 5 & 5 & 5 & 5 & 5 & 5 & 5 \\
\hline - Measurement method standard, valid and reliable & 3 & 3 & 3 & 3 & 3 & 3 & 3 & 3 & 3 & 3 \\
\hline - Exposure measured equally in all study groups & 3 & 0 & 3 & 0 & 0 & 0 & 0 & 3 & 0 & 0 \\
\hline 5. Outcome measures & 20 & 15 & 15 & 15 & 13 & 20 & 13 & 13 & 13 & 9 \\
\hline - Primary/secondary outcomes clearly defined & 5 & 5 & 5 & 5 & 5 & 5 & 5 & 5 & 5 & 5 \\
\hline - Outcomes assessed blind to exposure or intervention & 5 & 0 & 1 & 0 & 0 & 5 & 0 & 0 & 0 & 0 \\
\hline $\begin{array}{l}\text { - Method of outcome assessment standard, valid and } \\
\text { reliable }\end{array}$ & 5 & 5 & 4 & 5 & 5 & 5 & 5 & 5 & 3 & 4 \\
\hline - Length of follow-up adequate for question & 5 & 5 & 5 & 5 & 3 & 5 & 3 & 3 & 5 & 0 \\
\hline 6. Statistical Analysis & 19 & 3 & 15 & 11 & 8 & 5 & 9 & 6 & 7 & 7 \\
\hline - Statistical tests appropriate & 5 & 3 & 5 & 5 & 5 & 5 & 5 & 3 & 5 & 5 \\
\hline - Multiple comparisons taken into consideration & 3 & 0 & 3 & 3 & 3 & 0 & 3 & 3 & 2 & 2 \\
\hline - Modeling and multivariate techniques appropriate & 2 & 0 & 2 & 0 & 0 & 0 & 1 & 0 & 0 & 0 \\
\hline - Power calculation provided & 2 & 0 & 2 & 0 & 0 & 0 & 0 & 0 & 0 & 0 \\
\hline -Assessment of confounding & 5 & 0 & 3 & 3 & 0 & 0 & 0 & 0 & 0 & 0 \\
\hline - Dose-response assessment if appropriate & 2 & 0 & 0 & 0 & 0 & 0 & 0 & 0 & 0 & 0 \\
\hline 7. Results & 8 & 8 & 8 & 7 & 6 & 5 & 5 & 7 & 8 & 8 \\
\hline $\begin{array}{l}\text { - Measure of effect for outcomes and appropriate } \\
\text { measure of precision }\end{array}$ & 5 & 5 & 5 & 4 & 4 & 2 & 5 & 5 & 5 & 5 \\
\hline - Adequacy of follow-up for each study group & 3 & 3 & 3 & 3 & 2 & 3 & 0 & 2 & 3 & 3 \\
\hline 8. Discussion & 5 & 5 & 5 & 5 & 5 & 3 & 2 & 5 & 4 & 5 \\
\hline $\begin{array}{l}\text { - Conclusions supported by results with possible biases } \\
\text { and limitations taken into consideration }\end{array}$ & & 5 & 5 & 5 & 5 & 3 & 2 & 5 & 4 & 5 \\
\hline 9. Funding or Sponsorship & 5 & 0 & 5 & 0 & 5 & 0 & 0 & 0 & 0 & 0 \\
\hline - Type and sources of support for study & & 0 & 5 & 0 & 5 & 0 & 0 & 0 & 0 & 0 \\
\hline TOTAL SCORE $=$ & 100 & 49 & 85 & 58 & 63 & 52 & 49 & 61 & 52 & 49 \\
\hline
\end{tabular}


Pain Physician: January/February 2009:12:207-232

Table 8 cont.. Methodological assessment of observational studies evaluating the effectiveness of TAPS.

\begin{tabular}{|c|c|c|c|c|c|c|c|c|c|c|}
\hline CRITERION & $\begin{array}{l}\text { Weighted } \\
\text { Score } \\
\text { (points) }\end{array}$ & $\begin{array}{l}\text { Endres } \\
\text { et al } \\
(106)\end{array}$ & $\begin{array}{l}\text { Ergun } \\
\text { et al } \\
(101)\end{array}$ & $\begin{array}{l}\text { Finch } \\
\text { et al } \\
(42)\end{array}$ & $\begin{array}{l}\text { Freedman } \\
\text { et al (88) }\end{array}$ & $\begin{array}{l}\text { Gerszten } \\
\text { et al (83) }\end{array}$ & $\begin{array}{l}\text { Kapural } \\
\text { et al } \\
(95)\end{array}$ & $\begin{array}{l}\text { Kapural } \\
\text { et al } \\
(96)\end{array}$ & $\begin{array}{l}\text { Kapural } \\
\text { et al } \\
(99)\end{array}$ & $\begin{array}{c}\text { Kapural } \\
(100)\end{array}$ \\
\hline 1. Study Question & 2 & 2 & 2 & 2 & 2 & 2 & 2 & 2 & 2 & 2 \\
\hline - Clearly focused and appropriate question & & 2 & 2 & 2 & 2 & 2 & 2 & 2 & 2 & 2 \\
\hline 2. Study Population & 8 & 4 & 5 & 5 & 5 & 5 & 5 & 5 & 6 & 6 \\
\hline - Description of study population & 5 & 4 & 5 & 5 & 5 & 5 & 5 & 5 & 5 & 5 \\
\hline - Sample size justification & 3 & 0 & 0 & 0 & 0 & 0 & 0 & 0 & 1 & 1 \\
\hline $\begin{array}{l}\text { 3. Comparability of Subjects for All Observa- } \\
\text { tional Studies }\end{array}$ & 22 & 5 & 5 & 19 & 5 & 4 & 17 & 21 & 5 & 5 \\
\hline $\begin{array}{l}\text { - Specific inclusion/exclusion criteria for } \\
\text { all groups }\end{array}$ & 5 & 0 & 0 & 5 & 0 & 4 & 5 & 4 & 5 & 5 \\
\hline - Criteria applied equally to all groups & 3 & 0 & 0 & 3 & 0 & 0 & 3 & 3 & 0 & 0 \\
\hline $\begin{array}{l}\text { - Study groups comparable to non-partici- } \\
\text { pants with regard to confounding factors }\end{array}$ & 3 & 0 & 0 & 0 & 0 & 0 & 0 & 3 & 0 & 0 \\
\hline - Use of concurrent controls & 5 & 0 & 0 & 5 & 0 & 0 & 3 & 5 & 0 & 0 \\
\hline 4. Exposure or Intervention & 11 & 8 & 8 & 11 & 8 & 8 & 11 & 10 & 8 & 8 \\
\hline - Clear definition of exposure & 5 & 5 & 5 & 5 & 5 & 5 & 5 & 5 & 5 & 5 \\
\hline $\begin{array}{l}\text { - Measurement method standard, valid and } \\
\text { reliable }\end{array}$ & 3 & 3 & 3 & 3 & 3 & 3 & 3 & 2 & 3 & 3 \\
\hline $\begin{array}{l}\text { - Exposure measured equally in all study } \\
\text { groups }\end{array}$ & 3 & 0 & 0 & 3 & 0 & 0 & 3 & 3 & 0 & 0 \\
\hline 5. Outcome measures & 20 & 8 & 15 & 15 & 15 & 13 & 14 & 15 & 11 & 11 \\
\hline $\begin{array}{l}\text { - Primary/secondary outcomes clearly } \\
\text { defined }\end{array}$ & 5 & 5 & 5 & 5 & 5 & 3 & 5 & 5 & 5 & 5 \\
\hline $\begin{array}{l}\text { - Outcomes assessed blind to exposure or } \\
\text { intervention }\end{array}$ & 5 & 0 & 0 & 0 & 0 & 0 & 0 & 2 & 0 & 0 \\
\hline $\begin{array}{l}\text { - Method of outcome assessment standard, } \\
\text { valid and reliable }\end{array}$ & 5 & 3 & 5 & 5 & 5 & 5 & 5 & 5 & 5 & 3 \\
\hline - Length of follow-up adequate for question & 5 & 0 & 5 & 5 & 5 & 5 & 4 & 3 & 1 & 3 \\
\hline 6. Statistical Analysis & 19 & 5 & 9 & 4 & 13 & 5 & 13 & 16 & 8 & 8 \\
\hline - Statistical tests appropriate & 5 & 0 & 5 & 0 & 5 & 2 & 5 & 5 & 5 & 5 \\
\hline $\begin{array}{l}\text { - Multiple comparisons taken into } \\
\text { consideration }\end{array}$ & 3 & 0 & 2 & 0 & 3 & 3 & 3 & 2 & 3 & 3 \\
\hline $\begin{array}{l}\text { - Modeling and multivariate techniques } \\
\text { appropriate }\end{array}$ & 2 & 0 & 2 & 0 & 0 & 0 & 0 & 2 & 0 & 0 \\
\hline - Power calculation provided & 2 & 0 & 0 & 0 & 0 & 0 & 0 & 2 & 0 & 0 \\
\hline - Assessment of confounding & 5 & 0 & 0 & 0 & 5 & 0 & 5 & 5 & 0 & 0 \\
\hline - Dose-response assessment if appropriate & 2 & 0 & 0 & 0 & 0 & 0 & 0 & 0 & 0 & 0 \\
\hline 7. Results & 8 & 5 & 8 & 8 & 8 & 5 & 7 & 7 & 6 & 8 \\
\hline $\begin{array}{l}\text { - Measure of effect for outcomes and ap- } \\
\text { propriate measure of precision }\end{array}$ & 5 & 5 & 5 & 5 & 5 & 3 & 25 & 5 & 5 & 5 \\
\hline $\begin{array}{l}\text { - Adequacy of follow-up for each study } \\
\text { group }\end{array}$ & 3 & 0 & 3 & 3 & 3 & 2 & 2 & 2 & 1 & 3 \\
\hline 8. Discussion & 5 & 5 & 4 & 5 & 5 & 3 & 5 & 5 & 5 & 5 \\
\hline $\begin{array}{l}\text { - Conclusions supported by results with } \\
\text { possible biases and limitations taken into } \\
\text { consideration }\end{array}$ & & 5 & 4 & 0 & 5 & 3 & 5 & 5 & 5 & 5 \\
\hline 9. Funding or Sponsorship & 5 & 0 & 0 & 0 & 5 & 5 & 0 & 0 & 5 & 5 \\
\hline - Type and sources of support for study & & 0 & 0 & 0 & 5 & 5 & 5 & 0 & 5 & 5 \\
\hline TOTAL SCORE $=$ & 100 & 42 & 56 & 69 & 66 & 50 & 74 & 81 & 56 & 58 \\
\hline
\end{tabular}


Thermal Annular Procedures

Table 8 cont. Methodological assessment of observational studies evaluating the effectiveness of TAPS.

\begin{tabular}{|c|c|c|c|c|c|c|c|c|c|}
\hline CRITERION & $\begin{array}{c}\text { Weighted } \\
\text { Score } \\
\text { (points) }\end{array}$ & $\begin{array}{l}\text { Lee } \\
\text { et al } \\
(92)\end{array}$ & $\begin{array}{l}\text { Lutz } \\
\text { et al } \\
(91)\end{array}$ & $\begin{array}{c}\text { Maurer } \\
\text { and Squil- } \\
\text { lante (104) }\end{array}$ & $\begin{array}{l}\text { Maurer } \\
\text { et al (97) }\end{array}$ & $\begin{array}{l}\text { Mekhail } \\
\text { and } \\
\text { Kapural } \\
(94)\end{array}$ & $\begin{array}{c}\text { Nunley } \\
\text { et al } \\
(98)\end{array}$ & $\begin{array}{l}\text { Park } \\
\text { et al } \\
(113)\end{array}$ & $\begin{array}{l}\text { Saal and Saal } \\
(41,89,103)\end{array}$ \\
\hline 1. Study Question & 2 & 2 & 2 & 2 & 2 & 2 & 2 & 2 & 2 \\
\hline - Clearly focused and appropriate question & & 2 & 2 & 2 & 2 & 2 & 2 & 2 & 2 \\
\hline 2. Study Population & 8 & 5 & 5 & 5 & 5 & 5 & 5 & 5 & 5 \\
\hline - Description of study population & 5 & 5 & 5 & 5 & 5 & 5 & 5 & 5 & 5 \\
\hline - Sample size justification & 3 & 0 & 0 & 0 & 0 & 0 & 0 & 0 & 0 \\
\hline 3. Comparability of Subjects for All Observational Studies & 22 & 5 & 5 & 5 & 5 & 5 & 5 & 4 & 5 \\
\hline - Specific inclusion/exclusion criteria for all groups & 5 & 0 & 5 & 5 & 5 & 5 & 5 & 0 & 5 \\
\hline - Criteria applied equally to all groups & 3 & 0 & 0 & 0 & 0 & 0 & 0 & 0 & 0 \\
\hline $\begin{array}{l}\text { - Comparability of groups at baseline with regard to } \\
\text { disease status and prognostic factors }\end{array}$ & 3 & 0 & 0 & 0 & 0 & 0 & 0 & 0 & 0 \\
\hline $\begin{array}{l}\text { - Study groups comparable to non-participants with } \\
\text { regard to confounding factors }\end{array}$ & 3 & 0 & 0 & 0 & 0 & 0 & 0 & 0 & 0 \\
\hline - Use of concurrent controls & 5 & 0 & 0 & 0 & 0 & 0 & 0 & 0 & 0 \\
\hline $\begin{array}{l}\text { - Comparability of follow-up among groups at each } \\
\text { assessment }\end{array}$ & 3 & 0 & 0 & 0 & 0 & 0 & 0 & 0 & 0 \\
\hline 4. Exposure or Intervention & 11 & 8 & 8 & 7 & 8 & 8 & 8 & 7 & 8 \\
\hline - Clear definition of exposure & 5 & 5 & 5 & 4 & 5 & 5 & 5 & 5 & 5 \\
\hline - Measurement method standard, valid and reliable & 3 & 3 & 3 & 3 & 3 & 3 & 3 & 2 & 3 \\
\hline - Exposure measured equally in all study groups & 3 & 0 & 0 & 0 & 0 & 0 & 0 & 0 & 0 \\
\hline 5. Outcome measures & 20 & 15 & 14 & 13 & 15 & 13 & 13 & 13 & 15 \\
\hline - Primary/secondary outcomes clearly defined & 5 & 5 & 5 & 4 & 5 & 5 & 5 & 5 & 5 \\
\hline - Outcomes assessed blind to exposure or intervention & 5 & 0 & 0 & 0 & 0 & 0 & 0 & 0 & 0 \\
\hline $\begin{array}{l}\text { - Method of outcome assessment standard, valid and } \\
\text { reliable }\end{array}$ & 5 & 5 & 5 & 5 & 5 & 5 & 5 & 5 & 5 \\
\hline - Length of follow-up adequate for question & 5 & 5 & 4 & 4 & 5 & 3 & 3 & 3 & 5 \\
\hline 6. Statistical Analysis & 19 & 8 & 11 & 3 & 9 & 13 & 15 & 0 & 5 \\
\hline - Statistical tests appropriate & 5 & 5 & 5 & 3 & 5 & 5 & 5 & 0 & 5 \\
\hline - Multiple comparisons taken into consideration & 3 & 3 & 3 & 0 & 3 & 3 & 3 & 0 & 0 \\
\hline - Modeling and multivariate techniques appropriate & 2 & 0 & 0 & 0 & 1 & 0 & 2 & 0 & 0 \\
\hline - Power calculation provided & 2 & 0 & 0 & 0 & 0 & 0 & 0 & 0 & 0 \\
\hline - Assessment of confounding & 5 & 0 & 3 & 0 & 0 & 5 & 5 & 0 & 0 \\
\hline - Dose-response assessment if appropriate & 2 & 0 & 0 & 0 & 0 & 0 & 0 & 0 & 0 \\
\hline 7. Results & 8 & 5 & 8 & 7 & 8 & 7 & 7 & 6 & 8 \\
\hline $\begin{array}{l}\text { - Measure of effect for outcomes and appropriate mea- } \\
\text { sure of precision }\end{array}$ & 5 & 5 & 5 & 5 & 5 & 5 & 5 & 4 & 5 \\
\hline - Adequacy of follow-up for each study group & 3 & 0 & 3 & 2 & 3 & 2 & 2 & 2 & 3 \\
\hline 8. Discussion & 5 & 5 & 5 & 3 & 5 & 5 & 5 & 3 & 4 \\
\hline $\begin{array}{l}\text { - Conclusions supported by results with possible biases } \\
\text { and limitations taken into consideration }\end{array}$ & & 5 & 5 & 3 & 5 & 5 & 5 & 3 & 4 \\
\hline 9. Funding or Sponsorship & 5 & 0 & 0 & 0 & 5 & 0 & 0 & 0 & 0 \\
\hline - Type and sources of support for study & & 0 & 0 & 0 & 5 & 0 & 0 & 0 & 0 \\
\hline TOTAL SCORE= & 100 & 53 & 58 & 45 & 62 & 58 & 60 & 40 & 52 \\
\hline
\end{tabular}


Pain Physician: January/February 2009:12:207-232

Table 8 cont.. Methodological assessment of observational studies evaluating the effectiveness of TAPS.

\begin{tabular}{|c|c|c|c|c|c|c|}
\hline CRITERION & $\begin{array}{c}\text { Weighted } \\
\text { Score } \\
\text { (points) }\end{array}$ & Singh (110) & $\begin{array}{l}\text { Spruit and } \\
\text { Jacobs (111) }\end{array}$ & $\begin{array}{l}\text { Thompson } \\
\text { and Eckel } \\
\text { (112) }\end{array}$ & $\begin{array}{l}\text { Webster et al } \\
\quad(93)\end{array}$ & $\begin{array}{l}\text { Welch et al } \\
\quad(107)\end{array}$ \\
\hline 1. Study Question & 2 & 2 & 2 & 2 & 2 & 2 \\
\hline - Clearly focused and appropriate question & & 2 & 2 & 2 & 2 & 2 \\
\hline 2. Study Population & 8 & 5 & 5 & 5 & 3 & 3 \\
\hline - Description of study population & 5 & 5 & 4 & 5 & 3 & 3 \\
\hline - Sample size justification & 3 & 0 & 1 & 0 & 0 & 0 \\
\hline 3. Comparability of Subjects for All Observational Studies & 22 & 5 & 3 & 0 & 3 & 4 \\
\hline - Specific inclusion/exclusion criteria for all groups & 5 & 5 & 3 & 0 & 3 & 4 \\
\hline - Criteria applied equally to all groups & 3 & 0 & 0 & 0 & 0 & 0 \\
\hline $\begin{array}{l}\text { - Comparability of groups at baseline with regard to disease } \\
\text { status and prognostic factors }\end{array}$ & 3 & 0 & 0 & 0 & 0 & 0 \\
\hline $\begin{array}{l}\text { - Study groups comparable to non-participants with regard to } \\
\text { confounding factors }\end{array}$ & 3 & 0 & 0 & 0 & 0 & 0 \\
\hline - Use of concurrent controls & 5 & 0 & 0 & 0 & 0 & 0 \\
\hline - Comparability of follow-up among groups at each assessment & 3 & 0 & 0 & 0 & 0 & 0 \\
\hline 4. Exposure or Intervention & 11 & 8 & 8 & 3 & 3 & 8 \\
\hline - Clear definition of exposure & 5 & 5 & 5 & 3 & 1 & 5 \\
\hline - Measurement method standard, valid and reliable & 3 & 3 & 3 & 0 & 2 & 3 \\
\hline - Exposure measured equally in all study groups & 3 & 0 & 0 & 0 & 0 & 0 \\
\hline 5. Outcome measures & 20 & 10 & 11 & 12 & 8 & 9 \\
\hline - Primary/secondary outcomes clearly defined & 5 & 5 & 3 & 5 & 2 & 4 \\
\hline - Outcomes assessed blind to exposure or intervention & 5 & 0 & 0 & 0 & 0 & 0 \\
\hline - Method of outcome assessment standard, valid and reliable & 5 & 3 & 5 & 5 & 1 & 5 \\
\hline - Length of follow-up adequate for question & 5 & 2 & 3 & 2 & 5 & 0 \\
\hline 6. Statistical Analysis & 19 & 5 & 5 & 3 & 10 & 3 \\
\hline - Statistical tests appropriate & 5 & 0 & 5 & 3 & 5 & 3 \\
\hline - Multiple comparisons taken into consideration & 3 & 0 & 0 & 0 & 3 & 0 \\
\hline - Modeling and multivariate techniques appropriate & 2 & 0 & 0 & 0 & 0 & 0 \\
\hline - Power calculation provided & 2 & 0 & 0 & 0 & 0 & 0 \\
\hline - Assessment of confounding & 5 & 0 & 0 & 0 & 2 & 0 \\
\hline - Dose-response assessment if appropriate & 2 & 0 & 0 & 0 & 0 & 0 \\
\hline 7. Results & 8 & 7 & 4 & 5 & 5 & 3 \\
\hline $\begin{array}{l}\text { - Measure of effect for outcomes and appropriate measure of } \\
\text { precision }\end{array}$ & 5 & 5 & 4 & 2 & 2 & 0 \\
\hline - Adequacy of follow-up for each study group & 3 & 2 & 0 & 3 & 3 & 0 \\
\hline 8. Discussion & 5 & 5 & 3 & 2 & 5 & 3 \\
\hline $\begin{array}{l}\text { - Conclusions supported by results with possible biases and } \\
\text { limitations taken into consideration }\end{array}$ & & 5 & 3 & 2 & 5 & 0 \\
\hline 9. Funding or Sponsorship & 5 & 0 & 0 & 5 & 0 & 0 \\
\hline - Type and sources of support for study & & 0 & 0 & 5 & 0 & 0 \\
\hline TOTAL SCORE $=$ & 100 & 47 & 41 & 37 & 39 & 35 \\
\hline
\end{tabular}


the methodologic quality assessment criteria for evidence synthesis and 11 studies scored below 50 $(93,102,104-107,109-113)$, thus, they were not included in evidence synthesis.

\section{Study Characteristics}

Study characteristics for RCTs are illustrated in Table 9, whereas study characteristics of observational studies are illustrated in Table 10.

Table 9. Description of randomized controlled trials.

\begin{tabular}{|c|c|c|c|c|c|c|}
\hline Study/Methods & Participants & Inclusion/Exclusion & Interventions & Outcomes & Results & \begin{tabular}{|c|} 
Conclusion \\
Short-term $\leq$ \\
12 mos. \\
Long-term $>$ \\
12 mos.
\end{tabular} \\
\hline $\begin{array}{l}\text { Pauza et al } 2004 \\
(80) \\
\text { Randomized, } \\
\text { placebo-controlled, } \\
\text { double-blind, } \\
\text { prospective trial. } \\
\text { Study spon- } \\
\text { sored by device } \\
\text { manufacturer. }\end{array}$ & $\begin{array}{l}64 \text { patients Evaluated } \\
1360 \text { patients between } \\
\text { September } 2000 \text { and } \\
\text { April } 2002 ; 260 \text { poten- } \\
\text { tially met the criteria. } \\
\text { Study was done in a } \\
\text { private practice setting. } \\
\text { Of the } 37 \text { treated pa- } \\
\text { tients, } 32 \text { were included } \\
\text { in the analysis; of the } 27 \\
\text { sham patients, } 24 \text { were } \\
\text { included in the analysis. } \\
\\
\text { Pauza et al were unable } \\
\text { to enroll enough patients } \\
\text { to fully power his study } \\
\text { at } 80 \% \text {,study was statisti- } \\
\text { cally significant at } 60 \% .\end{array}$ & $\begin{array}{l}\text { Inclusion: age } 18-65 \\
\text { years; low back pain } \\
>\text { leg pain of }>6 \\
\text { months duration; } \\
\text { failure to improve after } \\
\text { nonoperative therapy; } \\
\text { no surgery within the } \\
\text { last three months; less } \\
\text { than } 20 \% \text { loss of disc } \\
\text { height. } \\
\text { Exclusion: abnormal } \\
\text { neurological exam; } \\
\text { Workers' Compensa- } \\
\text { tion; personal injury } \\
\text { litigation or receiving } \\
\text { disability. } \\
\text { Positive discography } \\
\text { and posterior annular } \\
\text { tears on CT scan. }\end{array}$ & $\begin{array}{l}\text { IDET } \\
37 \text { had IDET; } 27 \\
\text { had a sham proce- } \\
\text { dure in which the } \\
\text { introducer needle } \\
\text { was advanced to } \\
\text { the outer annulus, } \\
\text { but no catheter } \\
\text { placed. Sham pa- } \\
\text { tients were exposed } \\
\text { to a fluoroscopic } \\
\text { monitor show- } \\
\text { ing passage of the } \\
\text { electrode, with } \\
\text { appropriate sounds } \\
\text { during the putative } \\
\text { procedure. }\end{array}$ & $\begin{array}{l}\text { SF-36 and } \\
\text { VAS } \\
\text { Un- } \\
\text { blinded at } \\
\text { 6-months }\end{array}$ & $\begin{array}{l}56 \% \text { of the IDET } \\
\text { group had a } \\
\text { greater than } 2.0 \\
\text { improvement in } \\
\text { the VAS; } 38 \% \text { of } \\
\text { the sham group } \\
\text { did. } 24 \% \text { of the } \\
\text { treated group } \\
\text { had greater than } \\
75 \% \text { pain relief; } \\
4 \% \text { of the sham } \\
\text { group did. } \\
\text { The improve- } \\
\text { ment in the } \\
\text { IDET group was } \\
\text { significantly } \\
\text { better than the } \\
\text { sham. } \\
40 \% \text { of patients } \\
\text { treated with } \\
\text { IDET obtained } \\
50 \% \text { relief at } 6 \\
\text { months. }\end{array}$ & $\begin{array}{l}\text { Positive } \\
\text { short-term. } \\
\text { A needed- } \\
\text { to-treat } \\
\text { value of } 5 \\
\text { for achiev- } \\
\text { ing } 75 \% \text { re- } \\
\text { lief indicates } \\
\text { that it is a } \\
\text { worthwhile } \\
\text { intervention } \\
\text { for some } \\
\text { highly select } \\
\text { patients. }\end{array}$ \\
\hline $\begin{array}{l}\text { Freeman et al } 2005 \\
(79) \\
\text { Randomized, pla- } \\
\text { cebo- controlled, } \\
\text { double-blind, } \\
\text { prospective trial. } \\
\text { Study spon- } \\
\text { sored by device } \\
\text { manufacturer. }\end{array}$ & $\begin{array}{l}57 \text { subjects from } 3 \text { spine } \\
\text { practices in Australia. } \\
\text { Unable to enroll the } \\
75 \text { patients required to } \\
\text { power study at } 80 \% \text {. } \\
\text { Number of patients } \\
\text { screened to enroll the } 57 \\
\text { was not given. } \\
\text { Patients enrolled from } \\
\text { November } 1999 \text { to } \\
\text { December } 2001 . \\
\text { Between } 84 \% \text { and } 89 \% \\
\text { of enrollees had abnor- } \\
\text { mal reflexes. } 13 \% \text { of the } \\
\text { treated and } 5 \text { percent } \\
\text { of the sham patients } \\
\text { had positive Waddell } \\
\text { signs. Ten percent of the } \\
\text { treated group was on } \\
\text { disability. } \\
\text { Duration of low back } \\
\text { pain was up to } 20 \text { years }\end{array}$ & $\begin{array}{l}\text { Inclusion: symp- } \\
\text { toms of degenerative } \\
\text { lumbar disc disease > } \\
3 \text { months; failure to } \\
\text { improve with at least } 6 \\
\text { weeks of conservative } \\
\text { treatment; MRI docu- } \\
\text { mented degenerative } \\
\text { disease; one or } 2 \\
\text { positive levels on dis- } \\
\text { cography; dye spread } \\
\text { on post discography } \\
\text { CT scan to or beyond } \\
\text { the outer annulus; age } \\
>18 . \\
\text { Exclusion: loss of } \\
\text { more than } 50 \% \text { disc } \\
\text { height; severely dis- } \\
\text { rupted disc; } 3 \text { or more } \\
\text { symptomatic lumbar } \\
\text { discs; previous back } \\
\text { surgery; current injury } \\
\text { litigation. }\end{array}$ & $\begin{array}{l}\text { IDET } \\
\text { Treated group had } \\
\text { IDET, with catheter } \\
\text { covering at least } \\
75 \% \text { of the annular } \\
\text { tear. } \\
\text { The control had a } \\
\text { catheter placed in } \\
\text { the annulus and the } \\
\text { cable attached to it. } \\
\text { The cable was then } \\
\text { passed to an inde- } \\
\text { pendent technician } \\
\text { who would either } \\
\text { attach or not attach } \\
\text { the cable to the } \\
\text { IDET generator. } \\
\\
100 \text { mg of cefazolin } \\
\text { injected at end of } \\
\text { procedure. }\end{array}$ & $\begin{array}{l}\text { VAS, Low } \\
\text { Back Pain } \\
\text { Outcome } \\
\text { Score, } \\
\text { Oswestry } \\
\text { Disability } \\
\text { Index, SF- } \\
\text { 36, Zung } \\
\text { Depres- } \\
\text { sion Index } \\
\text { and the } \\
\text { Modified } \\
\text { Somatic } \\
\text { Perception } \\
\text { Question- } \\
\text { naire. }\end{array}$ & $\begin{array}{l}\text { At six months, } \\
\text { neither group } \\
\text { showed any } \\
\text { benefit in any } \\
\text { parameter. }\end{array}$ & $\begin{array}{l}\text { Negative } \\
\text { short-term }\end{array}$ \\
\hline
\end{tabular}


Table 10. Description of observational studies of TAPs.

\begin{tabular}{|c|c|c|c|c|c|c|}
\hline Study/Methods & Participants & Inclusion/Exclusion & Interventions & Outcomes & Results & $\begin{array}{c}\text { Conclusion } \\
\text { Short-term } \leq \\
12 \text { mos. } \\
\text { Long-term }> \\
12 \text { mos. }\end{array}$ \\
\hline $\begin{array}{l}\text { Bogduk and } \\
\text { Karasek 2000, } 2002 \\
(90,115) \\
\text { Prospective obser- } \\
\text { vational study, with } \\
\text { controls }\end{array}$ & $\begin{array}{l}53 \text { consecu- } \\
\text { tive patients } \\
\text { seen in private } \\
\text { pain practice } \\
\text { between May } \\
1998 \text { and No- } \\
\text { vember } 1998\end{array}$ & $\begin{array}{l}\text { Inclusion: Positive discogra- } \\
\text { phy at one to two levels, intact } \\
\text { annulus. } \\
\text { Disc height } \geq 80 \% \text { of normal. } \\
\text { Exclusion: Disc prolapsed, } \\
\text { neurologic disease, tumor, or } \\
\text { infection. }\end{array}$ & $\begin{array}{l}\text { Patients assigned to } \\
\text { treatment or control by } \\
\text { whether insurance autho- } \\
\text { rized procedure. } \\
\text { Catheter placed around } \\
\text { entire posterior an- } \\
\text { nulus. } 1 \mathrm{mg} \text { of cefazolin } \\
\text { injected intradiscally after } \\
\text { procedure } \\
\text { Control group given PT. }\end{array}$ & $\begin{array}{l}\text { Visual } \\
\text { Analogue } \\
\text { Scale (VAS), } \\
\text { return to } \\
\text { work and } \\
\text { opioid use }\end{array}$ & $\begin{array}{l}\text { Mean treated VAS } \\
\text { decreased from } 8.0 \\
\text { to } 3.0 \text { at } 2 \text { years; } 57 \% \\
\text { of treated group had } \\
50 \% \text { relief. }\end{array}$ & $\begin{array}{l}\text { Positive for } \\
\text { short- and } \\
\text { long-term } \\
\text { relief. } \\
\text { Powered at } \\
76 \% \text { at } 2 \text { years. }\end{array}$ \\
\hline $\begin{array}{l}\text { Gerszten at al } 2002 \\
(83) \\
\text { Prospective obser- } \\
\text { vational study }\end{array}$ & $\begin{array}{l}23 \text { consecutive } \\
\text { patients } \\
19 \text { patients } \\
\text { were on } \\
\text { Workers } \\
\text { Compensation. }\end{array}$ & $\begin{array}{l}\text { Inclusion criteria: back pain } \\
>6 \text { months duration. Low } \\
\text { back pain > leg pain; pain } \\
\text { with axial loading and relief } \\
\text { with recumbency; discogenic } \\
\text { disease on MRI or positive } \\
\text { discography; failure of conser- } \\
\text { vative treatment. }\end{array}$ & $\begin{array}{l}\text { IDET with catheter cover- } \\
\text { ing symptomatic side. No } \\
\text { antibiotics given. } \\
\text { Co-interventions were } \\
\text { limited to therapies given } \\
\text { prior to the IDET. }\end{array}$ & $\begin{array}{l}\text { Oswestry } \\
\text { Low Back } \\
\text { Pain Disabil- } \\
\text { ity and the } \\
\text { Short Form } \\
\text { (SF)-36 }\end{array}$ & $\begin{array}{l}47 \% \text { of patients had } \\
\text { significant ( }>7 \\
\text { points) improvement } \\
\text { in SF-36 scales. } 75 \% \\
\text { had improvement in } \\
\text { Oswestry. Work- } \\
\text { ers' Compensation } \\
\text { did not influence } \\
\text { outcome. }\end{array}$ & $\begin{array}{l}\text { Positive for } \\
\text { short- and } \\
\text { long-term } \\
\text { relief. }\end{array}$ \\
\hline $\begin{array}{l}\text { Saal and Saal } 2000 \\
\& 2002(41,89,103) \\
\text { Prospective obser- } \\
\text { vational study }\end{array}$ & $\begin{array}{l}53 \text { patients } \\
\text { selected from } \\
1,162 \text { low back } \\
\text { pain patients. } \\
34 \% \text { Workers' } \\
\text { Compensation. }\end{array}$ & $\begin{array}{l}\text { Inclusion: Low back pain }>6 \\
\text { months duration; Failure to } \\
\text { improve with non-operative } \\
\text { care; positive discography; } \\
\text { normal neurological exam; no } \\
\text { compressive lesion on MRI; } \\
\text { positive discography at }<1.25 \\
\text { mL of dye, maximum } 3 \text { levels } \\
\text { with negative control. }\end{array}$ & $\begin{array}{l}\text { IDET passed "as far as } \\
\text { possible around posterior } \\
\text { annulus. } 2-20 \text { mg of ce- } \\
\text { fazolin injected. No other } \\
\text { medications injected into } \\
\text { the disc. }\end{array}$ & $\begin{array}{l}\text { VAS, sitting } \\
\text { tolerance } \\
\text { and SF-36 }\end{array}$ & $\begin{array}{l}\text { At } 24 \text { months, at least } \\
72 \% \text { experienced } \\
\text { at least a } 2 \text { point } \\
\text { decrease in VAS and } \\
50 \% \text { had a } 4 \text { point } \\
\text { reduction. } 78 \% \text { had at } \\
\text { least a } 7 \text { point reduc- } \\
\text { tion in the bodily pain } \\
\text { scale of the SF-36. } \\
\text { Sitting tolerance in- } \\
\text { creased from a mean } \\
\text { of } 32 \text { to } 85 \text { minutes. } \\
97 \% \text { of the private pay } \\
\text { and } 83 \% \text { of the Work- } \\
\text { ers' Compensation } \\
\text { returned to work. }\end{array}$ & $\begin{array}{l}\text { Positive for } \\
\text { short- and } \\
\text { long-term } \\
\text { results. } \\
\text { Patients } \\
\text { with chronic } \\
\text { discogenic } \\
\text { low back pain } \\
\text { show sustained } \\
\text { improvement } \\
\text { in VAS, sitting } \\
\text { tolerance and } \\
\text { SF-36. }\end{array}$ \\
\hline $\begin{array}{l}\text { Cohen et al } 2003 \\
\text { (85) } \\
\text { Retrospective } \\
\text { observational pilot } \\
\text { study }\end{array}$ & $\begin{array}{l}70 \text { patients } \\
\text { with discogenic } \\
\text { low back pain. }\end{array}$ & $\begin{array}{l}\text { Inclusion criteria: Abnormal } \\
\text { MRI and positive discography. } \\
\text { Annular tears were permitted. } \\
\text { Low back pain }>6 \text { months } \\
\text { duration; age }<60 \text {; loss of } \\
\text { disc height }<50 \% \text {; failure } \\
\text { to respond to conservative } \\
\text { therapy; absence of prominent } \\
\text { radicular signs and symptoms. }\end{array}$ & $\begin{array}{l}\text { IDET limited to } 1 \text { or } \\
2 \text { discs. Coverage of at } \\
\text { least } 70 \% \text { of the posterior } \\
\text { annulus. Cefazolin and } \\
\text { bupivacaine, dose not } \\
\text { recorded, injected. }\end{array}$ & $\begin{array}{l}50 \% \text { reduc- } \\
\text { tion in pain } \\
\text { at } 6 \text {-months. }\end{array}$ & $\begin{array}{l}48 \% \text { had }>50 \% \text { relief. } \\
54 \% \text { of the nonobese } \\
\text { vs. } 10 \% \text { of the obese } \\
\text { had a good outcome; } \\
50 \% \text { of } 1 \text {-level vs. } 38 \% \\
\text { of } 2 \text {-level patients } \\
\text { had good outcomes. } \\
\text { No difference with } \\
\text { smoking, diabetes, } \\
\text { non-dermatomal leg } \\
\text { pain, and previous } \\
\text { surgery. }\end{array}$ & $\begin{array}{l}\text { Positive short- } \\
\text { term results. } \\
\text { Long-term } \\
\text { results not } \\
\text { available. }\end{array}$ \\
\hline $\begin{array}{l}\text { Freedman et al } \\
2002(88) \\
\text { Retrospective ob- } \\
\text { servational study, } \\
\text { no control }\end{array}$ & $\begin{array}{l}41 \text { active duty } \\
\text { soldiers seen } \\
\text { at Walter Reed } \\
\text { between } 1999 \\
\text { and } 2001 .\end{array}$ & $\begin{array}{l}\text { Inclusion: Low back pain > } \\
6 \text { months duration; positive } \\
\text { discography with at least one } \\
\text { normal disc; MRI absence } \\
\text { of nerve root compression, } \\
\text { tumor, infection or trauma; } \\
\text { no radicular symptoms; failed } \\
\text { nonoperative treatment }\end{array}$ & $\begin{array}{l}\text { IDET "using the protocol } \\
\text { described by Saal and } \\
\text { Saal." }\end{array}$ & $\begin{array}{l}50 \% \text { reduc- } \\
\text { tion in pain }\end{array}$ & $\begin{array}{l}29 \% \text { reported symp- } \\
\text { toms as improved at } \\
\text { last follow-up. Overall } \\
\text { satisfaction was } 16 \% \text {. } \\
52 \% \text { had a } 2 \text { point } \\
\text { reduction in VAS. }\end{array}$ & $\begin{array}{l}\text { Positive short- } \\
\text { term and nega- } \\
\text { tive long-term } \\
\text { outcomes. }\end{array}$ \\
\hline $\begin{array}{l}\text { Lee et al } 2003(92) \\
\text { Prospective obser- } \\
\text { vational study }\end{array}$ & $\begin{array}{l}62 \text { consecutive } \\
\text { patients. } \\
51 \text { patients } \\
\text { were available } \\
\text { for follow-up at } \\
2 \text { years } \\
20 \text { patients } \\
\text { were Workers' } \\
\text { Compensation } \\
\text { or no fault } \\
\text { insurance. }\end{array}$ & $\begin{array}{l}\text { Inclusion criteria: Low back } \\
\text { pain > } 6 \text { months; sitting> } \\
\text { standing pain; normal neuro- } \\
\text { logic exam; failure of conser- } \\
\text { vative care; no compressive } \\
\text { lesion on imaging; positive } \\
\text { discogram with annular tear; } \\
<50 \% \text { disc height. }\end{array}$ & $\begin{array}{l}\text { VAS, Roland Morris, and } \\
\text { NASS patient satisfaction } \\
\text { index. } \\
\text { 2-year follow-up }\end{array}$ & $\begin{array}{l}\text { IDET } \\
\text { catheter } \\
\text { passed "past } \\
\text { midline." No } \\
\text { mention of } \\
\text { intradiscal } \\
\text { antibiotics }\end{array}$ & $\begin{array}{l}53 \% \text { had VAS and } \\
\text { RM improvements > } \\
2 \text { points. } \\
\text { No difference with } \\
\text { age, insurance } \\
\text { (including Workers' } \\
\text { Compensation), } \\
\text { pre-IDET VAS, } \\
\text { number of levels, or } \\
\text { microdiscectomy. }\end{array}$ & $\begin{array}{l}\text { Positive short- } \\
\text { and long-term } \\
\text { results. }\end{array}$ \\
\hline
\end{tabular}


Table 10 cont. Description of observational studies of TAPs.

\begin{tabular}{|c|c|c|c|c|c|c|}
\hline Study/Methods & Participants & Inclusion/Exclusion & Interventions & Outcomes & Results & $\begin{array}{l}\text { Conclusion } \\
\text { Short-term } \leq \\
12 \text { mos. } \\
\text { Long-term }> \\
12 \text { mos. }\end{array}$ \\
\hline $\begin{array}{l}\text { Lutz et al } 2003 \text { (91) } \\
\text { Prospective obser- } \\
\text { vational study }\end{array}$ & $\begin{array}{l}33 \text { patients in } \\
\text { an academic- } \\
\text { affiliated pri- } \\
\text { vate physiatry } \\
\text { practice. } \\
\text { Dates of re- } \\
\text { cruitment not } \\
\text { given. }\end{array}$ & $\begin{array}{l}\text { Inclusion criteria: Low back } \\
\text { pain }>6 \text { months duration; } \\
\text { positive discography; non-re- } \\
\text { sponsive to conservative care. } \\
\text { Exclusion: }>50 \% \text { loss of disc } \\
\text { height; }>5 \mathrm{~mm} \text { disc extrusion } \\
\text { or sequestration; severe steno- } \\
\text { sis; spondylolisthesis; previous } \\
\text { spinal surgery; segmental } \\
\text { instability; infection }\end{array}$ & $\begin{array}{l}\text { VAS, Roland Morris, and } \\
\text { NASS patient satisfaction } \\
\text { index. } \\
\text { Success was a } 2 \text { point } \\
\text { improvement in VAS or } \\
\text { RM and a positive NASS } \\
\text { satisfaction response. } \\
\text { Follow-up at } 15 \text { months }\end{array}$ & $\begin{array}{l}\text { IDET } \\
\text { Catheter } \\
\text { "into the } \\
\text { posterior } \\
\text { annular wall } \\
\text { past the } \\
\text { midline." }\end{array}$ & $\begin{array}{l}\text { Mean change in } \\
\text { VAS was 3.9. 77\% } \\
\text { indicated they would } \\
\text { repeat the procedure. } \\
\text { Complete relief in } \\
24 \% \text { of patients and } \\
\text { partial relief in } 46 \% . \\
15 \% \text { of patients } \\
\text { required an epidural } \\
\text { steroid injection for } \\
\text { flare-up of leg pain. }\end{array}$ & $\begin{array}{l}\text { Positive short- } \\
\text { and long-term } \\
\text { results. }\end{array}$ \\
\hline $\begin{array}{l}\text { Davis et al } 2004 \\
\text { (114) } \\
\text { Retrospective ob- } \\
\text { servational study }\end{array}$ & $\begin{array}{l}60 \text { patients } \\
\text { referred from } \\
17 \text { spine spe- } \\
\text { cialists. IDET } \\
\text { performed by } 4 \\
\text { physicians. } \\
73 \% \text { of patients } \\
\text { responded to } \\
\text { questionnaire. }\end{array}$ & $\begin{array}{l}\text { Inclusion criteria: diagnosis } \\
\text { of discogenic low back pain }> \\
6 \text { months; positive discogram } \\
\text { with provocation discography } \\
\text { using }<2.5 \mathrm{ml} \text { of contrast, } \\
\text { with annular fissure; disc } \\
\text { height }>50 \% \text {; failed conserva- } \\
\text { tive therapy. }\end{array}$ & $\begin{array}{l}\text { Short and long question- } \\
\text { naires from the National } \\
\text { Low Back Pain Study. } \\
\text { Core questions were } \\
\text { pain intensity, functional } \\
\text { limitation, work status, } \\
\text { analgesic use, other treat- } \\
\text { ment for low back pain, } \\
\text { overall satisfaction. }\end{array}$ & $\begin{array}{l}\text { IDET. } \\
\text { Tech- } \\
\text { nique not } \\
\text { described. }\end{array}$ & $\begin{array}{l}37 \% \text { of patients had a } \\
\text { successful outcome. } \\
14 \% \text { had further } \\
\text { surgery at one year. } \\
\text { At two years, } 4 \text { more } \\
\text { patients had had } \\
\text { surgery. } \\
\text { One patient devel- } \\
\text { oped discitis and one } \\
\text { developed a Grade } \\
\text { I spondylolisthesis } \\
\text { requiring surgery. }\end{array}$ & $\begin{array}{l}\text { Negative } \\
\text { short- and } \\
\text { long-term } \\
\text { relief. }\end{array}$ \\
\hline $\begin{array}{l}\text { Derby et al } 2004 \\
\text { (86) } \\
\text { Retrospective pilot } \\
\text { study }\end{array}$ & $\begin{array}{l}35 \text { patients } \\
\text { for restorative } \\
\text { injection } \\
\text { therapy and } 74 \\
\text { for IDET. } \\
\text { "Retrospective- } \\
\text { ly performed } \\
\text { through the } \\
\text { analysis of a } \\
\text { prospectively } \\
\text { collected data } \\
\text { base." } \\
\text { Patients seen } \\
\text { between Janu- } \\
\text { ary } 2000 \text { and } \\
\text { October } 2002 .\end{array}$ & $\begin{array}{l}\text { Inclusion criteria: Chronic } \\
\text { low back pain not responsive } \\
\text { to conservative therapy; being } \\
\text { considered for additional sur- } \\
\text { gery; positive discography. } \\
\text { Prior surgery and, for the } \\
\text { injection group, prior IDET at } \\
\text { the treated level, was allowed. } \\
\text { For IDET, no focal neuro- } \\
\text { logical signs; single level; disc } \\
\text { height }>50 \% \text {. }\end{array}$ & $\begin{array}{l}\text { Compared effectiveness } \\
\text { of restorative injection } \\
\text { therapy and IDET. } \\
\text { VAS } \\
\text { Follow-up } 15.5 \text { months in } \\
\text { IDET and } 7.7 \text { months for } \\
\text { injection group. }\end{array}$ & $\begin{array}{l}\text { For } \\
\text { injection, } \\
\text { chondroitin } \\
\text { sulfate, glu- } \\
\text { cosamine, } \\
\text { DMSO, } \\
\text { bupivacaine, } \\
1-2 \mathrm{~mL} \\
\text { injected. } \\
\text { For IDET, } \\
\text { coverage of } \\
\text { entire poste- } \\
\text { rior annulus. } \\
\text { Cefazolin } \\
\text { (dose not } \\
\text { recorded) } \\
\text { injected } \\
\text { at end of } \\
\text { procedure. }\end{array}$ & $\begin{array}{l}\text { Mean improvement } \\
\text { for IDET was } 1.27 \\
\text { on VAS, versus } 2.2 \\
\text { for injection group. } \\
47.8 \% \text { of IDET group } \\
\text { felt better; } 65.5 \% \text { of } \\
\text { injection group did. } \\
\text { Pain relief was statisti- } \\
\text { cally significant for } \\
\text { both groups. } \\
81 \% \text { of injection } \\
\text { group had flare-up } \\
\text { compared to } 60 \% \text { of } \\
\text { IDET. Duration of } \\
\text { flare was } 8.6 \text { days for } \\
\text { injection group and } \\
33.1 \text { days for IDET. }\end{array}$ & $\begin{array}{l}\text { Positive short- } \\
\text { term relief. } \\
\text { Both IDET } \\
\text { and injection } \\
\text { therapy pro- } \\
\text { vided benefit. } \\
\\
\text { Results } \\
\text { subsumed } \\
\text { under Derby } \\
\text { et al (87) as } \\
\text { same patient } \\
\text { population } \\
\text { presumed to } \\
\text { be evaluated. }\end{array}$ \\
\hline $\begin{array}{l}\text { Derby et al } 2004 \\
\text { (87) } \\
\text { Retrospective ob- } \\
\text { servational study }\end{array}$ & $\begin{array}{l}99 \text { patients } \\
\text { seen in a } \\
\text { single practice } \\
\text { between } \\
\text { January } 1999 \\
\text { and December } \\
2000 \text { who did } \\
\text { not have subse- } \\
\text { quent surgery } \\
\text { and who met } \\
\text { inclusion } \\
\text { criteria. } \\
\text { Study assessed } \\
\text { changes in } \\
\text { referred leg } \\
\text { pain. }\end{array}$ & $\begin{array}{l}\text { Inclusion criteria: low back } \\
\text { or low back and leg pain }>6 \\
\text { months duration unrespon- } \\
\text { sive to conservative treatment; } \\
\text { negative straight leg raising; } \\
\text { non-focal neurological signs; } \\
\text { no compressive lesions on } \\
\text { MRI; disc protrusion }<2 \text { mm; } \\
\text { positive discogram with annu- } \\
\text { lar tear: no previous surgery; } \\
\text { disc height }>50 \% \text {. }\end{array}$ & $\begin{array}{l}\text { IDET with catheter cover- } \\
\text { age of the entire posterior } \\
\text { annulus. } \\
\text { 18-month follow-up. }\end{array}$ & $\begin{array}{l}\text { VAS and } \\
\text { 5-point } \\
\text { pain scale } \\
\text { from the } \\
\text { NASS low } \\
\text { back pain } \\
\text { assessment } \\
\text { instrument. } \\
\text { Patients } \\
\text { divided } \\
\text { into groups } \\
\text { of leg pain } \\
\text { dominant; } \\
\text { back pain } \\
\text { dominant; } \\
\text { leg and back } \\
\text { pain the } \\
\text { same. }\end{array}$ & $\begin{array}{l}52 \% \text { had an improve- } \\
\text { ment in leg pain, with } \\
\text { a mean improvement } \\
\text { of } 1.9 \text { (5 point scale). } \\
\text { Back pain decreased } \\
\text { from } 3.37 \text { to } 2.59 \\
(5 \text { point scale }= \\
\Delta 1.56 / 10) . \text { Relief of } \\
\text { back pain correlated } \\
\text { with relief of leg pain. }\end{array}$ & $\begin{array}{l}\text { Positive short- } \\
\text { and long-term } \\
\text { relief. } \\
\text { IDET can re- } \\
\text { lieve associated } \\
\text { limb pain. }\end{array}$ \\
\hline $\begin{array}{l}\text { Mekhail and } \\
\text { Kapural } 2004 \text { (94) } \\
\text { Prospective obser- } \\
\text { vational study }\end{array}$ & $\begin{array}{l}34 \text { consecutive } \\
\text { patients in an } \\
\text { academic pain } \\
\text { practice. } \\
32 \text { followed for } \\
1 \text { year. } \\
10 \text { patients } \\
\text { Workers } \\
\text { Compensation. }\end{array}$ & $\begin{array}{l}\text { Inclusion criteria: Disc height } \\
>50 \% \text {; no lumbar stenosis; } \\
\text { 1-or 2-level DDD; no disc } \\
\text { herniation on MRI; positive } \\
\text { discography; no psychological } \\
\text { issues. }\end{array}$ & $\begin{array}{l}\text { IDET } \\
\text { Catheter position not } \\
\text { described. }\end{array}$ & $\begin{array}{l}\text { Pain dis- } \\
\text { ability index } \\
\text { (7 different } \\
\text { activities of } \\
\text { daily living } \\
\text { plus VAS) } \\
\text { Follow-up 1 } \\
\text { year. }\end{array}$ & $\begin{array}{l}\text { Non-Workers' } \\
\text { Compensation had a } \\
78 \% \text { decrease in VAS } \\
\text { versus 53\% for Work- } \\
\text { ers' Compensation. } \\
\text { No significant dif- } \\
\text { ference in gender, } \\
\text { smoking or age. }\end{array}$ & $\begin{array}{l}\text { Positive short- } \\
\text { and long-term } \\
\text { relief. }\end{array}$ \\
\hline
\end{tabular}


Table 10 cont. Description of observational studies of TAPs.

\begin{tabular}{|c|c|c|c|c|c|c|}
\hline Study/Methods & Participants & Inclusion/Exclusion & Interventions & Outcomes & Results & $\begin{array}{c}\text { Conclusion } \\
\text { Short-term } \leq \\
12 \text { mos. } \\
\text { Long-term }> \\
12 \text { mos. }\end{array}$ \\
\hline $\begin{array}{l}\text { Kapural et al } 2004 \\
(95) \\
\text { Prospective obser- } \\
\text { vational study }\end{array}$ & $\begin{array}{l}17 \text { consecu- } \\
\text { tive patients } \\
\text { with multilevel } \\
\text { disc disease } \\
\text { matched } \\
\text { with } 17 \text { of } 22 \\
\text { consecutive } \\
\text { patients with } \\
1-\text { or } 2 \text {-level } \\
\text { disc disease. }\end{array}$ & $\begin{array}{l}\text { Inclusion criteria: Low back } \\
\text { pain }>6 \text { months not responsive } \\
\text { to conservative therapy; no } \\
\text { compressive radiculopathy; no } \\
\text { previous surgery at symptom- } \\
\text { atic levels; disc height }>50 \% \text {; } \\
\text { no signs or symptoms of } \\
\text { stenosis; positive discography. }\end{array}$ & $\begin{array}{l}\text { IDET } \\
\text { Catheter position not } \\
\text { described. }\end{array}$ & $\begin{array}{l}\text { Pain dis- } \\
\text { ability index } \\
\text { ( } 7 \text { different } \\
\text { activities of } \\
\text { daily living } \\
\text { plus VAS) } \\
\text { Follow-up } 1 \\
\text { year. }\end{array}$ & $\begin{array}{l}\text { The } 1 \text { - or } 2 \text { level } \\
\text { group had a pretreat- } \\
\text { ment VAS of } 7.7 \text { ver- } \\
\text { sus } 2.5 \text { at } 12 \text { months. } \\
\text { The multi-level group } \\
\text { decreased from } 7.4 \\
\text { to } 4.9 \text {. }\end{array}$ & $\begin{array}{l}\text { Positive short- } \\
\text { and long-term } \\
\text { relief. IDET } \\
\text { results are bet- } \\
\text { ter in patients } \\
\text { with 1- or } \\
\text { 2-level disc } \\
\text { disease. }\end{array}$ \\
\hline $\begin{array}{l}\text { Kapural et al } 2005 \\
(96) \\
\text { Prospective } \\
\text { controlled non- } \\
\text { randomized obser- } \\
\text { vational study }\end{array}$ & $\begin{array}{l}42 \text { matched } \\
\text { patients, } 21 \\
\text { with IDET } \\
\text { and } 21 \text { with } \\
\text { radiofrequency } \\
\text { annuloplasty } \\
\text { in an academic } \\
\text { pain practice. }\end{array}$ & $\begin{array}{l}\text { Inclusion criteria: Low back } \\
\text { pain }>6 \text { months not respon- } \\
\text { sive to conservative care; no } \\
\text { compressive radiculopathy; } \\
\text { positive discography; no prior } \\
\text { surgery; disc height > 50\%; } \\
\text { not Workers' Compensation } \\
\text { claimants }\end{array}$ & $\begin{array}{l}\text { IDET and radiofrequency } \\
\text { annuloplasty }\end{array}$ & $\begin{array}{l}\text { Pain dis- } \\
\text { ability index } \\
\text { question- } \\
\text { naire. } \\
12 \text { month } \\
\text { follow-up }\end{array}$ & $\begin{array}{l}\text { IDET VAS decreased } \\
\text { from } 7.4 \text { to } 1.4 \text {; } \\
\text { radiofrequency } \\
\text { annuloplasty VAS } \\
\text { decreased from } 6.6 \\
\text { to } 4.4 \\
\text { PDI scores mirrored } \\
\text { these changes. }\end{array}$ & $\begin{array}{l}\text { Positive short- } \\
\text { term for IDET. } \\
\text { Negative } \\
\text { long-term for } \\
\text { radiofrequency } \\
\text { annuloplasty }\end{array}$ \\
\hline $\begin{array}{l}\text { Finch et al } 2005 \\
(42) \\
\text { Prospective, } \\
\text { controlled but not } \\
\text { randomized, obser- } \\
\text { vational study }\end{array}$ & $\begin{array}{l}46 \text { patients: } \\
31 \text { treated; } 15 \\
\text { non-treated, } \\
\text { because of } \\
\text { insurance } \\
\text { denial, served } \\
\text { as control. } \\
\text { About } 2 / 3 \text { of } \\
\text { patients were } \\
\text { Workers' } \\
\text { Compensation }\end{array}$ & $\begin{array}{l}\text { Inclusion criteria: low back } \\
\text { pain }>6 \text { months duration not } \\
\text { responsive to conservative } \\
\text { care; positive discography } \\
\text { with annular tears; disc height } \\
>70 \% \text {. }\end{array}$ & $\begin{array}{l}\text { Radiofrequency } \\
\text { annuloplasty }\end{array}$ & $\begin{array}{l}\text { VAS, Os- } \\
\text { westry Dis- } \\
\text { ability Index, } \\
\text { Medication } \\
\text { Quantifica- } \\
\text { tion Score } \\
\text { 12-month } \\
\text { follow-up }\end{array}$ & $\begin{array}{l}\text { The treated group } \\
\text { had a } 37 \% \text { average } \\
\text { decrease in VAS. } \\
\text { Oswestry had a sig- } \\
\text { nificant decrease No } \\
\text { difference in outcome } \\
\text { based upon Workers' } \\
\text { Compensation status. }\end{array}$ & $\begin{array}{l}\text { Positive } \\
\text { long-term for } \\
\text { radiofrequency } \\
\text { annuloplasty } \\
\text { Negative } \\
\text { short-term } \\
\text { relief. }\end{array}$ \\
\hline $\begin{array}{l}\text { Bryce et al } 2005 \\
(84) \\
\text { Prospective obser- } \\
\text { vational study }\end{array}$ & $\begin{array}{l}86 \text { consecu- } \\
\text { tive patients } \\
\text { in a rural } \\
\text { Wisconsin pain } \\
\text { practice. }\end{array}$ & $\begin{array}{l}\text { Inclusion criteria: Low back } \\
\text { pain }>6 \text { months duration } \\
\text { unresponsive to conservative } \\
\text { treatment; back pain }>60 \% \\
\text { of other symptoms; normal } \\
\text { neurological exam; positive } \\
\text { discography; annular tears; } \\
\text { 18-50 years. }\end{array}$ & IDET & $\begin{array}{l}\text { VAS and } \\
\text { Roland Mor- } \\
\text { ris Disability } \\
\text { Question- } \\
\text { naire } \\
24 \text { months } \\
\text { follow-up }\end{array}$ & $\begin{array}{l}\text { Significant ( }>20 \\
\text { point) improvement } \\
\text { in RMDQ. } \\
\text { VAS improved. } \\
\text { Improvement best in } \\
\text { females and in those } \\
\text { aged } 18-45 \text { years. }\end{array}$ & $\begin{array}{l}\text { Positive short- } \\
\text { and long-term } \\
\text { relief. }\end{array}$ \\
\hline $\begin{array}{l}\text { Maurer et al } 2008 \\
(97) \\
\text { Prospective obser- } \\
\text { vational study }\end{array}$ & $\begin{array}{l}56 \text { consecutive } \\
\text { patients } \\
16 \% \text { of patients } \\
\text { on Workers' } \\
\text { Compensation } \\
\text { Industry spon- } \\
\text { sored study }\end{array}$ & $\begin{array}{l}\text { Inclusion criteria: low back } \\
\text { pain }>6 \text { months duration; } \\
\text { disc height }>50 \% \text {; normal } \\
\text { lower extremity neurological } \\
\text { exam; } 1-3 \text { desiccated discs } \\
\text { discography; and posterior } \\
\text { annular tear. Exclusion: previ- } \\
\text { ous back surgery. }\end{array}$ & IDET & $\begin{array}{l}\text { Back pain } \\
\text { sever- } \\
\text { ity, physical } \\
\text { function } \\
\text { and quality } \\
\text { of life } \\
\text { Follow-up } \\
24 \text { months }\end{array}$ & $\begin{array}{l}\text { VAS improved by } \\
61 \% \text {. There were } \\
\text { also significant } \\
\text { improvements in } \\
\text { sitting, standing and } \\
\text { walking tolerances. } \\
61 \% \text { improvement in } \\
\text { SF-36. } 75 \% \text { treatment } \\
\text { successes. }\end{array}$ & $\begin{array}{l}\text { Positive short- } \\
\text { and long-term } \\
\text { improvement. }\end{array}$ \\
\hline $\begin{array}{l}\text { Nunley et al } 2008 \\
\text { (98) } \\
\text { Prospective obser- } \\
\text { vational study }\end{array}$ & $\begin{array}{l}53 \text { consecu- } \\
\text { tive Workers' } \\
\text { Compensation } \\
\text { patients with } \\
\text { low back pain. }\end{array}$ & $\begin{array}{l}\text { Inclusion criteria: persistent } \\
\text { low back pain }>6 \text { months } \\
\text { with failure to respond to con- } \\
\text { servative therapy; prior spine } \\
\text { surgery; abnormal neurologi- } \\
\text { cal exam; disc height }>40 \% \text {; } \\
\text { positive discography with an } \\
\text { annular tear; BMI between } \\
20.1-44.2 \text {. }\end{array}$ & IDET & $\begin{array}{l}\text { VAS, Os- } \\
\text { westry and } \\
\text { self-assess- } \\
\text { ment ques- } \\
\text { tionnaires } \\
\text { of pain and } \\
\text { disability } \\
12 \text {-month } \\
\text { follow-up. }\end{array}$ & $\begin{array}{l}\text { The mean reduction } \\
\text { of VAS was } 62.6 \% \text {, } \\
\text { while the mean } \\
\text { reduction in Oswestry } \\
\text { was } 69.3 \% \text {. There } \\
\text { was no significant } \\
\text { effect of age or BMI } \\
\text { on outcome. Narcotic } \\
\text { use dropped from } \\
51 \% \text { initially to } 13.2 \% \\
\text { after treatment. } 47 \% \\
\text { returned to work in a } \\
\text { full or partial capacity }\end{array}$ & $\begin{array}{l}\text { Positive short- } \\
\text { and long-term } \\
\text { improvement. }\end{array}$ \\
\hline
\end{tabular}


Table 10 cont. Description of observational studies of TAPs.

\begin{tabular}{|c|c|c|c|c|c|c|}
\hline Study/Methods & Participants & Inclusion/Exclusion & Interventions & Outcomes & Results & $\begin{array}{l}\text { Conclusion } \\
\text { Short-term } \leq \\
12 \text { mos. } \\
\text { Long-term }> \\
12 \text { mos. }\end{array}$ \\
\hline $\begin{array}{l}\text { Ergun et al } 2008 \\
(101) \\
\text { Prospective obser- } \\
\text { vational study }\end{array}$ & $\begin{array}{l}39 \text { consecutive } \\
\text { patients in a } \\
\text { Turkish pain } \\
\text { practice. }\end{array}$ & $\begin{array}{l}\text { Inclusion criteria: Low back } \\
\text { pain }>6 \text { months non-respon- } \\
\text { sive to conservative therapy; } \\
\text { 1- or } 2 \text {-level disease; no evi- } \\
\text { dence of nerve root compres- } \\
\text { sion; }>50 \% \text { disc height. }\end{array}$ & $\begin{array}{l}\text { IDET } \\
\text { Catheter covered } 75 \% \text { of } \\
\text { the annulus. } \\
\text { No post procedure } \\
\text { antibiotics. }\end{array}$ & $\begin{array}{l}\text { Turkish } \\
\text { version of } \\
\text { the Oswestry } \\
\text { Disability } \\
\text { Index } \\
\text { 18-month } \\
\text { follow-up }\end{array}$ & $\begin{array}{l}\text { At } 18 \text { months, the } \\
\text { mean decrease in ODI } \\
\text { was } 24 . \\
79.5 \% \text { of patients } \\
\text { benefited. } \\
\text { No complications. }\end{array}$ & $\begin{array}{l}\text { Positive short- } \\
\text { and long-term } \\
\text { improvement. }\end{array}$ \\
\hline $\begin{array}{l}\text { Kapural et al } 2008 \\
\text { (99) and Kapural } \\
2008(100) \\
\text { Prospective pilot } \\
\text { observational study }\end{array}$ & $\begin{array}{l}15 \text { patients } \\
\text { aged } 22 \text { to } \\
55, \text { with } 13 \\
\text { available at } 12 \\
\text { months }\end{array}$ & $\begin{array}{l}\text { Inclusion criteria: low back } \\
\text { pain of greater than } 6 \text { months } \\
\text { duration, back pain greater } \\
\text { than leg pain, positive discog- } \\
\text { raphy, disc height greater than } \\
50 \% \text { of normal and one or two } \\
\text { level disc disease only; normal } \\
\text { weight; age } 55 \text { or less. }\end{array}$ & IDB & $\begin{array}{l}\text { VAS, Oswes- } \\
\text { try, SF-36 } \\
12 \text {-month } \\
\text { follow-up }\end{array}$ & $\begin{array}{l}\text { Seven of } 13 \text { patients } \\
\text { had more than } 50 \% \\
\text { pain relief. The VAS } \\
\text { decreased from } 7 \\
(6,8) \text { to } 4(1,6) \text { at } \\
12 \text {-months. } \\
\text { The Oswestry } \\
\text { decreased from } 23.3 \\
\text { to } 17.5 \text { and the SF- } 36 \\
\text { physical functioning } \\
\text { scores increased from } \\
51 \text { to } 67 \text {. }\end{array}$ & $\begin{array}{l}\text { Positive short- } \\
\text { and long-term } \\
\text { improvement. }\end{array}$ \\
\hline
\end{tabular}

\section{Intradiscal Electrothermal Therapy}

\section{Level of Evidence}

Table 11 illustrates results of published studies of effectiveness of IDET.

Two RCTs meeting inclusion criteria exist for IDET $(79,80)$. One was favorable, with a significant placebo effect, and one was non-responsive to the study question.

Sixteen observational studies meeting inclusion criteria evaluated IDET. Of these, 14 studies found the procedure to be effective $(83-85,87,89,91,92,94$ $98,101,115)$. Three studies found it to be ineffective $(86,88,114)$

The indicated evidence for IDET is Level II-2 based on U.S. Preventive Services Task Force (USPSTF) criteria.

\section{Recommendations}

Based upon one positive RCT and one unfavorable $\mathrm{RCT}$, which was not responsive to the study's null hypothesis, a recommendation of $2 \mathrm{~A} /$ weak recommendation is provided.

The recommendation is supplemented based on observational evidence derived from multiple studies.

\section{Radiofrequency Annuloplasty}

\section{Level of Evidence}

Table 12 illustrates the results of published studies of effectiveness of radiofrequency annuloplasty.

Two studies dealt with radiofrequency annuloplasty $(42,96)$. Finch et al $(42)$, in a case series, found the procedure to be effective. Kapural et al (96), in an observational study, found radiofrequency annuloplasty to be less effective than IDET.

The level of evidence for radiofrequency annuloplasty is II-3 (uncertain).

\section{Recommendations}

The recommendation is $2 \mathrm{C} /$ weak.

\section{Intradiscal Biacuplasty}

\section{Level of Evidence}

Table 13 illustrates the results of published studies of effectiveness of IDB.

Only one pilot study is available for IDB $(99,100)$ evaluating the effectiveness of the procedure. There is a randomized, placebo-controlled trial in progress.

The level of evidence for IDB is Level III. 
Table 11. Results of published studies of effectiveness of IDET.

\begin{tabular}{|c|c|c|c|c|c|c|c|}
\hline \multirow[b]{2}{*}{ Study } & \multirow[b]{2}{*}{$\begin{array}{c}\text { Study } \\
\text { Characteristics }\end{array}$} & \multirow[b]{2}{*}{$\begin{array}{l}\text { Methodological } \\
\text { Quality Scoring }\end{array}$} & \multirow[b]{2}{*}{ Participants } & \multicolumn{2}{|c|}{$\begin{array}{l}\text { Pain Relief } \\
\end{array}$} & \multicolumn{2}{|c|}{ Results } \\
\hline & & & & $\leq 12 \mathrm{mos}$ & $>12 \mathrm{mos}$. & $\begin{array}{c}\text { Short-term } \\
\text { relief } \leq 12 \\
\text { mos. }\end{array}$ & $\begin{array}{c}\text { Long-term } \\
\text { relief }>12 \\
\text { mos. }\end{array}$ \\
\hline $\begin{array}{l}\text { Pauza et al } 2004 \\
(80)\end{array}$ & RA & 68 & 64 & $\begin{array}{c}56 \% \text { had } 2 \text { point } \\
\text { decrease } \\
40 \% \text { had }>50 \% \\
\text { decrease }\end{array}$ & NA & Yes & NA \\
\hline $\begin{array}{l}\text { Freeman et al } \\
2005 \text { (79) }\end{array}$ & RA & 61 & 57 & No change & NA & No & NA \\
\hline $\begin{array}{l}\text { Karasek and } \\
\text { Bogduk 2000 and } \\
2002(90,115)\end{array}$ & $\mathrm{O}$ & 85 & 53 & $70 \%$ & $57 \%$ & Yes & Yes \\
\hline $\begin{array}{l}\text { Gerszten et al } \\
2002(83)\end{array}$ & $\mathrm{O}$ & 50 & 27 & $75 \%$ & $75 \%$ & Yes & Yes \\
\hline $\begin{array}{l}\text { Saal and Saal } \\
2000 \& 2002 \\
(41,89,103)\end{array}$ & $\mathrm{O}$ & 52 & 53 & SI & SI & Yes & Yes \\
\hline $\begin{array}{l}\text { Cohen et al } 2003 \\
(85)\end{array}$ & $\mathrm{O}$ & 80 & 70 & $48 \%$ & NA & Yes & NA \\
\hline $\begin{array}{l}\text { Freedman et al } \\
2002(88)\end{array}$ & $\mathrm{O}$ & 66 & 41 & $47 \%$ & $\begin{array}{c}16 \%>50 \% \\
\text { decrease }\end{array}$ & Yes & No \\
\hline Lee et al 2003 (92) & $\mathrm{O}$ & 53 & 62 & NA & $53 \%$ & Yes & Yes \\
\hline $\begin{array}{l}\text { Lutz et al } 2003 \\
\text { (91) }\end{array}$ & $\mathrm{O}$ & 58 & 33 & NA & $70 \%$ & Yes & Yes \\
\hline $\begin{array}{l}\text { Davis et al } 2004 \\
\text { (114) }\end{array}$ & $\mathrm{O}$ & 52 & 60 & NA & $37 \%$ & No & No \\
\hline $\begin{array}{l}\text { Derby et al } 2004 \\
\text { (86) }\end{array}$ & $\mathrm{O}$ & 61 & $\begin{array}{l}34 \text { Injection } \\
74 \text { IDET }\end{array}$ & $\begin{array}{c}2.2 \text { point } \\
\text { decrease for } \\
\text { injection } \\
1.27 \text { for IDET }\end{array}$ & NA & Yes & No \\
\hline $\begin{array}{l}\text { Derby et al } 2004 \\
\text { (87) }\end{array}$ & $\mathrm{O}$ & 52 & 99 & NA & $\begin{array}{c}52 \% \\
1.56 \text { point } \\
\text { decrease } \\
\text { back pain }\end{array}$ & Yes & Yes \\
\hline $\begin{array}{l}\text { Mekhail and } \\
\text { Kapural } 2004 \text { (94) }\end{array}$ & $\mathrm{O}$ & 58 & 34 & SI & SI & Yes & Yes \\
\hline $\begin{array}{l}\text { Kapural et al } 2004 \\
\text { (95) }\end{array}$ & $\mathrm{O}$ & 74 & 34 & SI & SI & Yes & Yes \\
\hline $\begin{array}{l}\text { Kapural et al } 2005 \\
\text { (96) }\end{array}$ & $\mathrm{O}$ & 81 & 21 & SI & SI & Yes & Yes \\
\hline $\begin{array}{l}\text { Bryce et al } 2005 \\
(84)\end{array}$ & $\mathrm{O}$ & 58 & 86 & SI & SI & Yes & Yes \\
\hline $\begin{array}{l}\text { Maurer et al } 2008 \\
\text { (97) }\end{array}$ & $\mathrm{O}$ & 62 & 56 & SI & SI & Yes & Yes \\
\hline $\begin{array}{l}\text { Nunley et al } 2008 \\
\text { (98) }\end{array}$ & $\mathrm{O}$ & 60 & 53 & SI & NA & Yes & NA \\
\hline $\begin{array}{l}\text { Ergun et al } 2008 \\
\text { (101) }\end{array}$ & $\mathrm{O}$ & 56 & 39 & NA & $79 \%$ & NA & Yes \\
\hline
\end{tabular}

$\mathrm{O}=$ observational; $\mathrm{RA}$ = randomized; VAS = visual analog scale; $\mathrm{SI}$ = significant improvement; NSI = no significant improvement; NA = not available 
Table 12. Results of published studies of effectiveness of radiofrequency annuloplasty.

\begin{tabular}{|c|c|c|c|c|c|c|c|}
\hline \multirow{2}{*}{ Study } & \multirow{2}{*}{$\begin{array}{c}\text { Study } \\
\text { Characteristics }\end{array}$} & \multirow{2}{*}{$\begin{array}{l}\text { Methodological } \\
\text { Quality Scoring }\end{array}$} & \multirow{2}{*}{ Participants } & \multicolumn{2}{|c|}{ Pain Relief (VAS) } & \multicolumn{2}{|c|}{ Results } \\
\hline & & & & $\leq 12$ mos. & $>12$ mos. & $\begin{array}{c}\text { Short-term } \\
\text { relief } \leq 12 \mathrm{mos}\end{array}$ & $\begin{array}{c}\text { Long-term } \\
\text { relief }>12 \\
\text { mos. }\end{array}$ \\
\hline $\begin{array}{l}\text { Finch et al } \\
2005(42)\end{array}$ & $\mathrm{O}$ & 69 & 46 & $37 \%$ & NA & No & NA \\
\hline $\begin{array}{l}\text { Kapural } \\
\text { et al } 2005 \\
\text { (96) }\end{array}$ & $\mathrm{O}$ & 81 & 21 & NSI & NA & No & NA \\
\hline
\end{tabular}

$\mathrm{O}=$ observational; $\mathrm{RA}$ = randomized; $\mathrm{NSI}$ = no significant improvement; $\mathrm{NA}=$ not available

Table 13. Results of published studies of effectiveness of IDB.

\begin{tabular}{|c|c|c|c|c|c|c|c|}
\hline \multirow{2}{*}{ Study } & \multirow{2}{*}{$\begin{array}{c}\text { Study } \\
\text { Characteristics }\end{array}$} & \multirow{2}{*}{$\begin{array}{l}\text { Methodological } \\
\text { Quality Scoring }\end{array}$} & \multirow{2}{*}{ Participants } & \multicolumn{2}{|c|}{ Pain Relief (VAS) } & \multicolumn{2}{|c|}{ Results } \\
\hline & & & & $\leq 12$ mos. & $>12 \mathrm{mos}$ & $\begin{array}{l}\text { Short-term relief } \\
\quad \leq 12 \text { mos. }\end{array}$ & $\begin{array}{l}\text { Long-term relief } \\
\quad>12 \text { mos. }\end{array}$ \\
\hline $\begin{array}{l}\text { Kapural } \\
\text { et al } 2008 \\
(99) \text { and } \\
\text { Kapural } \\
2008(100)\end{array}$ & $\mathrm{O}$ & $56 / 58$ & 15 & SI & SI & Yes & Yes \\
\hline
\end{tabular}

$\mathrm{O}=$ observational; $\mathrm{SI}=$ significant improvement

\section{Recommendations}

The recommendation is $2 \mathrm{C} /$ very weak.

\section{Discussion}

Three thermal annular procedures (TAPs) are currently available - IDET, radiofrequency annuloplasty, and IDB. IDET is supported with an indicated evidence of Level II-2 with a 2A/weak recommendation. Radiofrequency annuloplasty and IDB have a evidence levels of II-3 and III with 2C/very weak recommendations.

A meta-analysis of IDET was performed by Appleby et al (44). The lead author was an employee of the device manufacturer. He reviewed 17 reports on IDET, finding a mean improvement in VAS of 2.9. The mean improvement of the physical functioning scale of the SF-36 was 21.1 points. The mean improvement of the Oswestry Disability Index was 7.0 points. The complication rate was $0.8 \%$. Complications which resolved included burning pain in the legs, paresthesias, foot drop, headache, increased radicular pain, dural puncture, incontinence of bowel, and non-dermatomal leg pain. Discitis did appear in one patient. Patients did go on to fusion, although the incidence was not provided. Appleby et al (44) did note the importance of selection criteria. They reported that the pooled results of published studies provided compelling evidence of the relative efficacy and safety of IDET. Andersson et al (45) in a systematic review of spinal fusion and IDET concluded that the majority of patients reported improvement in symptoms following both spinal fusion and the IDET procedure. The IDET procedure appears to offer sufficiently similar symptom amelioration to spinal fusion without the attendant complications.

Two RCTs met the criteria for inclusion in this review, those of Freeman et al (79) and Pauza et al (80). Both evaluated IDET. Pauza et al's study (80) has been criticized for the extensive placebo effect. It has also been criticized for how highly selective it was in terms 
of screening patients, raising questions as whether the results could be extrapolated to clinical practice. Another criticism is that it did not control for patients with multilevel degenerative disease, raising the question as to whether, despite the rigorous selection process, patients who have been shown to not respond well to IDET, those with multilevel degenerative disease, were provided the procedure, thereby decreasing the apparent efficacy of the procedure. Placebo effects are a major source of patient response in RCTs. The concern is that there is additional relief above and beyond the placebo effect in the treated group. Pauza et al (80) have shown such an incremental effect; this analysis showing the NNT to get $75 \%$ relief (an endpoint which is arbitrarily chosen) convincingly argues for the efficacy of the procedure.

Freeman et al's study (79) is interesting for a number of reasons. Despite having enrolled patients earlier than Pauza et al (80), the study did not publish until after Pauza et al's paper was published (although Freeman et al did present the study at a meeting prior to publication of Pauza et al's study). Freeman et al opines that the clinical significance of the results found by Pauza were uncertain, although this statement is presented as a matter of fact, with no supporting documentation. The quality of blinding in Freeman et al's study is unclear. In Freeman et al's study, after the IDET catheter was placed, the randomization was done by a technician either connecting or not connecting the cable to the generator. In performing an IDET procedure, the generator makes a noise every 5 seconds. If the catheter is not attached, then the characteristic noise is not made. Freeman et al (79) do not disclose how this conundrum was handled: it is not clear whether the treating physician was therefore blinded. Procedurally, the $100 \mathrm{mg}$ of cefazolin is a very large dose and may have caused a chemical irritation to the disc. Further, despite the entrance criterion of a normal neurological examination, patients were admitted with an abnormal motor assessment and abnormal sensory exams and positive Waddell's findings. In addition, the inclusion criteria allowed patients with back pain of up to 20 years duration; it is not clear that patients with pain of so long standing would benefit from any procedure. Freeman et al also included Workers' Compensation patients. These are all potential confounding factors.

These criticisms pale, however, in light of the failure of any patients, either treated or placebo to respond. One review suggests that this finding is be- cause of the physical therapy provided prior to the procedure. This explanation is unlikely because all TAP studies had as an inclusion criteria failure to respond to conservative treatment, which would include physical therapy. For example, Webster et al (93), in a study critical of IDET, found that $100 \%$ of patients had physical therapy prior to IDET.

The inability to demonstrate any positive response to IDET in either group, in contradistinction to all other published reports, raises questions regarding study methodology. The absence of placebo response would seem to suggest an undefined methodological error in the study. One potential answer is that the study was inadequately powered. We know that the desired power was not met, but Freeman et al (79) does not disclose the actual power achieved. The presence of a placebo effect has been well studied and has been attributed to multiple factors, including the observation (Hawthorne) effect, the natural course of disease, and regression of measured observations to the mean (116-118). In addition, observer bias can influence outcomes (Pygmalion effect), although the mechanism by which participants become aware of latent bias is unknown (119). The absence of placebo effect has not been well evaluated. However, given the extensive body of literature documenting its presence, the absence of placebo effect must be interpreted as a serious methodological flaw in how Freeman et al's study (79) was conducted, although, as documented by the Pygmalion effect, the nature of this flaw is unknown. As such, the strongest conclusion that one can draw from Freeman et al's study is that it is non-responsive in evaluating the null hypothesis that IDET is no more effective than placebo for the treatment of chronic discogenic low back pain.

Twelve of 16 observational studies favored IDET. None of these studies is without flaw. Bodguk and Karasek's $(90,115)$ series does use a control group, but that control has a potential nocebo effect in that their insurance declined coverage of IDET. Regardless, they did find ITDA to provide significant relief in approximately one-half of the patients. Gerszten et al's (83) study was done in a neurosurgical setting, in which patients who were candidates for fusion were offered IDET if they could not or would not have a fusion. Seventy percent of their patients were Workers' Compensation. There is some question as to how the procedure performed with annular coverage limited to the symptomatic side(s) and one case of dural puncture. Using the Oswestry as an outcome measure, they 
found improvement in $75 \%$ of their cases.

Saal and Saal's (89) study showed significant improvement in VAS and SF-36 scores at 2 years after IDET. A significant confounding factor was their role as the developers of the device. Cohen et al (85), in a retrospective pilot study, investigated the risk factors for a poor outcome after IDET, finding only obesity to be associated with an adverse outcome. They did note that $50 \%$ of patients had a successful outcome, defined as a $50 \%$ reduction in pain. Freedman et al (88), like Cohen et al (85), looked at the military population. With the definition of success at $50 \%$ relief, only $16 \%$ had a successful outcome. Further, only $29 \%$ reported sustained relief at the latest follow-up and only $16 \%$ were either somewhat or very satisfied with their outcome. Freedman et al's study (88) is therefore considered a negative regarding the efficacy of IDET. However, $50 \%$ of patients had a sustained 2 point reduction in their VAS, a reduction which is felt to be clinically significant. Given the military active duty nature of the study population, it would be useful to have more information regarding what the soldiers would have required for them to be satisfied with the procedure. While Freedman et al does discuss the success and complication rate of IDET, they do not present analogous information regarding the satisfaction rate amongst active duty military with fusion. Regardless, Freedman et al do state that they would continue to offer IDET.

Lee et al (92) and Lutz et al (91) published IDET studies in 2003, both out of the same practice. These appear to be separate patient groups, although the methodological parameters are the same. Both found the procedure to be effective. They did focus on function and noted that some patients, including a professional ballerina and a college athlete, were able to return to full activity.

Davis et al's retrospective study (114) of the outcomes of IDET patients in the Los Angeles area found that the study was less effective than suggested by others. Their study is clouded by the use of high volume, $2.5 \mathrm{~mL}$, discography, suggesting that there may have been false positives on discography. They did include an undisclosed number of Workers' Compensation patients. A confounding factor is that of overuse of IDET; the absence of a Workers' Compensation fee schedule for IDET in the ambulatory setting during the period when patients were recruited created an incentive for over utilization. The outcome measure was the percentage of patients going on to fusion. Given that
IDET is designed to prevent some, but not all, patients from going to fusion, the relevance of this measure is unclear.

Derby et al (86) compared IDET and restorative injection therapy, finding that both caused clinically significant decreases in pain. The IDET group had only a 1.27 decrease in VAS, a decrease which does not met the 2 point VAS decrease criterion. The study suggests either that IDET operates by enhancing the hypermetabolic repair response of chondrocytes or that a strong placebo response was present in the study. Because of the failure to meet the 2 point VAS decrease threshold, this study was rated negative.

Derby et al (87) published a second study in 2004 looking at the same personal data base (not the Oratec National Registry), assessing the efficacy of IDET in relieving leg pain. Derby et al's two studies $(86,87)$, as they involve the same database, should be viewed as one when evaluating the effectiveness of IDET. The study is interesting in that it shows that non-dermatomal leg pain associated with disc disease can be relieved by IDET. Because it showed only a 1.56 point decrease in VAS, it is rated negative for IDET.

Kapural et al (95) in a 2004 prospective, controlled, but not randomized, study showed that IDET provided better results when patients had one or two level disease than when they had multilevel disease.

Mekhail and Kapural (94) published another prospective study in 2004 examining whether additional inclusion criteria improved outcomes. They found that while Workers' Compensation patients had less improvement in reported pain scores than patients covered under private insurance, there was no functional difference between the 2 groups. This finding raises the question as to whether the inclusion of Workers' Compensation patients in effectiveness studies biases the results.

Webster et al (93) performed an interesting review of a Workers' Compensation data base, finding that the inclusion criteria for IDET were not followed in $68 \%$ of the cases, suggesting poor patient selection. The study focused on the criteria that could be identified, including narcotic usage, whether the same provider did the discogram as did the procedure (provider self-referral), and return to work. The study did not have a vehicle for how many patients were able to avoid fusion; however, the study does highlight the role of Workers' Compensation status as being a confounding factor in evaluating IDET. The study is rated negative for IDET. 
Bryce et al's study (84) of IDET in a rural population documented positive results from the procedure. Interestingly, Bryce et al's study showed a gender preference for females. Ergun et al (101) evaluated the effectiveness of IDET in a group of Turkish patients. They used $2.5 \mathrm{~mL}$ of dye for discography, raising the possibility of false-positive discography results. Nunley et al (98) looked at the effects of IDET in Workers' Compensation patients. This study, which did not have a control, showed good results in this population, in contradistinction to other studies. It also included patients with a BMI of up to 44; again, this is in contradistinction to other studies showing worse outcomes with increased weight. Regardless, Nunley et al did find good outcomes with their patient population. Maurer et al (97) looked at the efficacy of IDET in patients treated in an academic orthopedic practice. Interestingly, although the patients were treated between 1998 and 2002, the paper was not published until 2008. The study was industry supported. Maurer et al did find IDET effective.

Regarding radiofrequency annuloplasty, Finch et al (42), who developed the radiofrequency annuloplasty technique, published a pilot study showing improvement in VAS and Oswestry. Kapural et al (96) performed a nonrandomized prospective study comparing IDET and radiofrequency annuloplasty. In this study, the IDET group showed significant improvement whereas the radiofrequency annuloplasty group did not.

The only IDB studies are Kapural et al's 2 reports $(99,100)$ on their pilot study. Although IDB appears to be an interesting technology, final assessment of this technology must be deferred until the IDB RCT is completed.

Complications of the procedure are uncommon and usually transient $(120,121)$. Not all patients will get relief and some patients will go on to have further spinal procedures. However, being able to avoid surgery in approximately $50 \%$ of patients, at minimal risk and cost to the patients, is the significant advantage of TAPs. Careful patient selection is critical to obtain maximum benefit from the procedure. The current understanding of the inclusion criteria would be low back pain of greater than 6 months duration non-responsive to conservative treatment; back pain greater than leg pain; positive well-performed discography with a negative control; presence of an annular tear; disc disease limited to one or 2 levels; disc height at least $50 \%$ of normal; no evidence of compressive radiculopathy or abnormal lower extremity neurological exam other than diminished ankle reflexes; disc bulges $\leq 5 \mathrm{~mm}$; no prior surgery at the treated level; no symptoms or signs of stenosis; no pending Workers' Compensation claims; and no significant depression or psychiatric issues on exam or history. Exclusion criteria would include tumor; systemic infection; localized infection at needle site; coagulopathy or unexplained bleeding; progressive neurological defects; history of substance abuse; manual labor; smoking; $\mathrm{BMI}>30$ or age $>55$. By these criteria, those who are categorically related to Medicare based upon age would not be candidates for IDET for failure to meet the age criterion. Those who are eligible for Medicare benefits for other reasons, such as disability, would have to be evaluated by the other criteria to determine potential eligibility for the procedure.

The mode of action is unclear. A likely candidate is neuroablation of nociceptors in the annulus, although this hypothesis is not entirely satisfactory in that pain relief does not occur immediately after the procedure. Other hypotheses, including enhancement of chondrocytes activity, exist and have not been disproven.

TAP have been extensively criticized for a variety of reasons. The procedure seems to have been politicized. It is speculation as to why this has occurred. One argument seems to be the rapid, even indiscriminate acceptance of the procedure, with many payors not seeing benefit because of poor patient selection and many practitioners feeling that promotion of the product was self-serving. Another argument would be that the procedure does prevent some patients from going on to a fusion, thereby raising financial concerns in certain quarters.

The present systematic review is limited in that the authors do perform TAP in their clinical practices; as such, they can be expected to have a bias in favor of the procedure. This potential bias can be minimized only by acknowledging its existence and presenting a methodology-transparent review, so that all can compare the authors' evaluation of the literature with their own.

This systematic review highlights the strengths and weaknesses of an interesting body of literature dealing with TAP. It is unlikely that there will be any further RCTs dealing with either IDET or radiofrequency annuloplasty. The data base that we currently have will serve as the basis for any future discussion. However, further studies are in progress for IDB. This review will require an update. 
Contemporary thought on clinical practice guidelines emphasize the need for consideration of patient preference $(122,123)$. TAPs provide patients with chronic discogenic low back pain who also meet the other treatment criteria an option which is supported by evidence-based medicine and which might meet their personal values and preferences. Further, TAP does meet the criteria of clinical relevance as described by Staal et al (59).

\section{Conclusion}

TAPs provide clinical benefit in about one-half or more of carefully selected patients. Some patients who have a TAP go on to fusion. TAPs are not a substitute for fusion and a patient does not need to have been offered a fusion prior to proceeding with a TAP. TAPs offer carefully selected patients the potential to get relief of their otherwise refractory low back pain. A diligent review of the evidence supports the use of TAPs.

\section{Acknowledgements}

The authors wish to thank the editorial board of Pain Physician for review and criticism in improving the manuscript; Sekar Edem and Vidyasagar Pampati, MSc, statistician, for their assistance in search of literature; and Tonie M. Hatton and Diane E. Neihoff, transcriptionists, for their assistance in the preparation of this manuscript.

\section{References}

1. Carey TS, Garrett JM, Jackman A, Hadler N. Recurrence and care seeking after acute back pain. Results of a long term follow up study. Med Care 1999; 37:157164.

2. Wahlgren DR, Atkinson JH, Epping-Jordan JE, Williams RA, Pruitt SD, Klapow JC, Patterson TL, Grant I, Webster JS, Slater MA. One-year follow up of first onset low back pain. Pain 1997; 73:213221.

3. Croft PR, Macfarlane GJ, Papageorgiou AC, Thomas E, Silman AJ. Outcome of low back pain in general practice: A prospective study. BMJ 1998; 316:13561359.

4. Elliott AM, Smith BH, Hannaford PC, Smith WC, Chambers WA. The course of chronic pain in the community: Results of a 4-year follow-up study. Pain 2002; 99:299-307.

5. Enthoven P, Skargern E, Oberg B. Clinical course in patients seeking primary care for back or neck pain: A prospective 5-year follow-up of outcome and health care consumption with subgroup analysis. Spine 2004; 29:2458-2465.

6. Cassidy JD, Côté P, Carroll LJ, Kristman V. Incidence and course of low back pain episodes in the general population. Spine 2005; 30:2817-2823.

7. Miedema HS, Chorus AM, Wevers CW, van der Linden S. Chronicity of back problems during working life. Spine 1998; 23:2021-2028.

8. Kuslich SD, Ulstrom CL, Michael CJ. The tissue origin of low back pain and sci- atica: A report of pain response to tissue stimulation during operation on the lumbar spine using local anesthesia. Orthop Clin North Am 1991; 22:181-187.

9. Schwarzer AC, Aprill CN, Derby R, Fortin 16 J, Kine G, Bogduk N. The prevalence and clinical features of internal disc disruption in patients with chronic low back pain. Spine 1995; 20:1878-1883.

10. Pang WW, Mok MS, Lin ML, Chang DP, 17 Hwang $\mathrm{MH}$. Application of spinal pain mapping in the diagnosis of low back pain - analysis of 104 cases. Acta Anaesthesiol Sin 1998; 36:71-74.

11. Manchikanti L, Singh V, Pampati V, Damron K, Barnhill R, Beyer C, Cash K. Evaluation of the relative contributions of various structures in chronic low back pain. Pain Physician 2001; 4:308-316.

12. Buenaventura RM, Shah RV, Patel V, Benyamin R, Singh V. Systematic review of discography as a diagnostic test for spinal pain: An update. Pain Physician 2007; 10:147-164.

13. Smith SE, Darden BV, Rhyne AL, Wood KE. Outcome of unoperated discogrampositive low back pain. Spine 1995; 20:1997-2001.

14. DeBerard, MS, Masters KS, Colledge AL, Schleusener RL, Schlegel JD. Outcomes of posterolateral lumbar fusion in Utah patients receiving workers' compensation: A retrospective cohort study. Spine 2001; 26:738-747.

15. Gilchrist RV, Slipman C. Clinical anato- 22 my of the lumbar spine. In Manchikanti L, Slipman CW, Fellows B (eds). Inter- ventional Pain Management: Low Back Pain - Diagnosis and Treatment. ASIPP Publishing, Paducah, KY, 2002, pp 4758.

Hadjipavlou AG, Tzermiadianos MN, Bogduk N, Zindrick MR. The pathophysiology of disc degeneration: A critical review. J Bone Joint Surg Br 2008; 90:1261-1270.

17. Rajasekaran S, Babu JN, Arun R, Armstrong BR, Shetty AP, Murugan S. ISSLS prize winner: A study of diffusion in human lumbar discs: A serial magnetic resonance imaging study documenting the influence of the endplate on diffusion in normal and degenerate discs. Spine 2004; 29:2654-2667.

8. Boos N, Weissbach S, Rohrbach H, Weiler C, Spratt KF, Nerlich AG. Classification of age-related changes in lumbar intervertebral discs: 2002 Volvo Award in basic science. Spine 2002; 27:26312644 .

19. Roberts S, Urban JP, Evans H, Eisenstein SM. Transport properties of the human cartilage endplate in relation to its composition and calcification. Spine 1996; 21:415-420.

20. Hurri H, Karppinen J. Discogenic pain. Pain 2004; 112:225-228.

21. Peng B, Wu W, Hou S, Li P, Zhang C, Yang Y. The pathogenesis of discogenic low back pain. J Bone Joint Surg Br 2005; 87:62-67.

2. Cavanaugh JM, Kallakuri S, Ozaktay AC. Innervation of the rabbit lumbar intervertebral disc and posterior longitu- 
dinal ligament. Spine 1995; 20:20802085.

23. Palmgren T, Gronblad M, Virri J, Kaapa E, Karaharju E. An immunohistochemical study of nerve structures in the anulus fibrosus of human normal lumbar intervertebral discs. Spine 1999; 24:2075-2079.

24. Groen GJ, Baljet B, Drukker J. Nerves and nerve plexuses of the human vertebral column. Am J Anat 1990; 188:282 396.

25. Bogduk N, Tynan W, Wilson AS. The nerve supply to the human lumbar intervertebral discs. J Anat 1981; 132:3956.

26. Cavanaugh JM, Ozaktay AC, Yamashita T, Avramov A, Getchell TV, King Al. Mechanisms of low back pain: A neurophysiologic and neuroanatomic study. Clin Orthop Relat Res 1997; 335:166180.

27. Roberts S, Eisenstein SM, Menage J, Evans EH, Ashton IK. Mechanoreceptors in intervertebral discs. Morphology, distribution, and neuropeptides. Spine 1995; 20:2645-2651.

28. Videman T, Nurminen M. The occurrence of anular tears and their relation to lifetime back pain history: A cadaveric study using barium sulfate discog raphy. Spine 2004; 29:2668-2676.

29. Coppes MH, Marani E, Thomeer RT, Groen GJ. Innervation of painful lumbar discs. Spine 1997; 22:2342-2349.

30. Ashton IK, Roberts S, Jaffray DC, Polak $J M$, Eisenstein SM. Neuropeptides in the human intervertebral disc. J Orthop Res 1994; 12:186-192.

31. Nakamura S, Takahashi K, Takahashi Y, Morinaga T, Shimada Y, Moriya H. Origin of nerves supplying the posterior portion of lumbar intervertebral discs in rats. Spine 1996; 21:917-924.

32. Ohtori S, Takahashi Y, Takahashi K, Yamagata M, Chiba T, Tanaka K, Hirayama J, Moriya H. Sensory innervation of the dorsal portion of the lumbar intervertebral disc in rats. Spine 1999; 24:2295-2299.

33. Fagan A, Moore R, Vernon Roberts B, Blumbergs $P$, Fraser R. The innervation of the intervertebral disc: A quantitative analysis. Spine 2003; 28:25702576.

34. Ohtori S, Takahashi K, Chiba T, Yamagata M, Sameda H, Moriya H. Sensory innervation of the dorsal portion of the lumbar intervertebral discs in rats. Spine 2001; 26:946-950.
35. McCarthy PW, Carruthers B, Martin D, Petts P. Immunohistochemical demonstration of sensory nerve fibers and endings in lumbar intervertebral discs of the rat. Spine 1991; 16:653-655.

36. Aoki Y, Ohtori S, Takahashi K, Ino H, Takahashi Y, Chiba T, Moriya H. Innervation of the lumbar intervertebral disc by nerve growth factor-dependent neurons related to inflammatory pain. Spine 2004; 29:1077-1081.

37. Burke JG, Watson RW, McCormack D, Dowling FE, Walsh MG, Fitzpatrick JM. Intervertebral discs which cause low back pain secrete high levels of proinflammatory mediators. J Bone Joint Surg Br 2002; 84:196-201.

38. Peng B, Hao J, Hou S, Wu W, Jiang D, Fu X, Yang Y. Possible pathogenesis of painful intervertebral disc degeneration. Spine 2006; 31:560-566.

39. Derby R, Baker RM, Lee CH, Anderson PA. Evidence-informed management of chronic low back pain with intradiscal electrothermal therapy. Spine J 2008; 8:80-95.

40. Kapural L, Mekhail N, Hicks D, Kapural M, Sloan S, Moghal N, Ross J, Petrinec D. Histological changes and temperature distribution studies of a novel bipolar radiofrequency heating system in degenerated and non-degenerated human cadaver lumbar discs. Pain Med 2008; 9:68-75.

41. Saal JS, Saal JA. Management of chronic discogenic low back pain with a thermal intradiscal catheter. A preliminary report. Spine 2000; 25:382-388.

42. Finch PM, Price LM, Drummond PD. Radiofrequency heating of painful annular disruptions: One-year outcomes. J Spinal Disord Tech 2005; 18:6-13.

43. Gibson JN, Waddell G. Surgery for degenerative lumbar spondylosis: Updated Cochrane review. Spine 2005; 30:2312-2320.

44. Appleby D, Andersson G, Totta M. Metaanalysis of the efficacy and safety of intradiscal electrothermal therapy (IDET). Pain Med 2006; 7:308-316.

45. Andersson GB, Mekhail NA, Block JE. Treatment of intractable discogenic low back pain. A systematic review of spinal fusion and intradiscal electrothermal therapy (IDET). Pain Physician 2006; 9:237-248.

46. Freeman BJ. IDET: A critical appraisal of the evidence. Eur Spine J 2006; 15:448457.

47. Freeman BJ, Mehdian R. Intradiscal electrothermal therapy, percutaneous discectomy, nucleoplasty: What is the current evidence? Curr Pain Headache Rep 2008; 12:14-21.

48. American College of Occupational and Environmental Medicine. Low Back Disorders Chapter. In: Occupational Medicine Practice Guidelines: Evaluation and Management of Common Health Problems and Functional Recovery of Workers, Second Edition. American College of Occupational and Environmental Medicine Press, Elk Grove Village, 2007.

49. Manchikanti L, Singh V, Derby R, Schultz DM, Benyamin RM, Prager JP, Hirsch JA. Reassessment of evidence synthesis of occupational medicine practice guidelines for interventional pain management. Pain Physician 2008; 11:393482.

50. Phurrough S, Salive M, O'Connor D, Schafer J. Decision Memo for Thermal Intradiscal Procedures. 2008 [cited September 30, 2008]. www.cms.hhs. $\mathrm{gov} / \mathrm{mcd} / \mathrm{viewdecisionmemo.asp?fro}$ $\mathrm{m} 2=$ viewdecisionmemo.asp\&id $=215$ \&

51. Koes BW, Scholten RJ, Mens JMA, Bouter LM. Efficacy of epidural steroid injections for low-back pain and sciatica: A systematic review of randomized clinical trials. Pain 1995; 63:279-288.

52. West S, King V, Carey TS, Lohr KN, McKoy N, Sutton SF, Lux L. Systems to Rate the Strength of Scientific Evidence, Evidence Report, Technology Assessment No. 47. AHRQ Publication No. 02E016. Rockville, MD: Agency for Healthcare Research and Quality, 2002. www. thecre.com/pdf/ahrq-system-strength. pdf

53. Atluri S, Datta S, Falco FJ, Lee M. Systematic review of diagnostic utility and therapeutic effectiveness of thoracic facet joint interventions. Pain Physician 2008; 11:611-629.

54. Conn A, Buenaventura R, Datta S, Abdi $S$, Diwan S. Systematic review of caudal epidurals injections in the management of chronic low back pain. Pain Physician 2009; 12:109-135.

55. Parr AT, Diwan S, Abdi S. Lumbar interlaminar epidural injections in managing chronic low back and lower extremity pain: A systematic review. Pain Physician 2009; 12:163-188.

56. Buenaventura R, Datta S, Abdi S, Smith HS. Systematic review of therapeutic lumbar transforaminal epidural steroid injections. Pain Physician 2009; 12:233-251. 
57. Benyamin R., Singh V, Parr AT, Conn A, Diwan S, Abdi S. Systematic review of the effectiveness of cervical epidurals in the management of chronic neck pain. Pain Physician 2009; 12:137-157.

58. van Tulder $M$, Furlan $A$, Bombardier C, Bouter L, Editorial Board of the Cochrane Collaboration Back Review Group. Updated method guidelines for systematic reviews in the Cochrane Collaboration Back Review Group. Spine 2003; 28:1290-1299.

59. Staal JB, de Bie R, de Vet HC, Hildebrandt J, Nelemans P. Injection therapy for subacute and chronic low-back pain. Cochrane Database Syst Rev 2008; 3:CDo01824.

60. Salaffi F, Stancati A, Silvestri CA, Ciapetti A, Grassi W. Minimal clinically important changes in chronic musculoskeletal pain intensity measured on a numerical rating scale. Eur I Pain 2004; 8:283-291.

61. Bombardier C. Outcome assessments in the evaluation of treatment of spinal disorders: Summary and general recommendations. Spine 2000; 25:31003103.

62. Manchikanti L, Boswell MV, Giordano J. Evidence-based interventional pain management: Principles, problems, potential and applications. Pain Physician 2007; 10:329-356.

63. Manchikanti L, Abdi S, Lucas LF. Evidence synthesis and development of guidelines in interventional pain management. Pain Physician 2005; 8:7386.

64. Manchikanti L, Heavner J, Racz GB, Mekhail NA, Schultz DM, Hansen HC, Singh V. Methods for evidence synthesis in interventional pain management. Pain Physician 2003; 6:89-111.

65. Manchikanti L. Evidence-based medicine, systematic reviews, and guidelines in interventional pain management: Part 1: Introduction and general considerations. Pain Physician 2008; 11:161-186.

66. Manchikanti L, Hirsch JA, Smith HS. Evidence-based medicine, systematic reviews, and guidelines in interventional pain management: Part 2: Randomized controlled trials. Pain Physician 2008; 11:717-773.

67. Manchikanti L, Smith HS, Hirsch JA. Evidence-based medicine, systematic reviews, and guidelines in interventional pain management: Part 3: Systematic reviews and meta-analysis of random- ized trials. Pain Physician 2009; 12:35 72.

68. Manchikanti L, Smith HS, Hirsch JA. Evidence-based medicine, systematic reviews, and guidelines in interventional pain management: Part 4: Observational studies. Pain Physician 2009; 12:73-108.

69. Farrar JT, Young JP Jr, LaMoreaux L, Werth JL, Poole RM. Clinical importance of changes in chronic pain intensity measured on an 11-point numerical pain rating scale. Pain 2001; 94:149158.

70. Manchikanti L, Singh V, Falco FJE, Cash KA, Pampati V. Effectiveness of thoracic medial branch blocks in managing chronic pain: A preliminary report of a randomized, double-blind controlled trial: Clinical trial NCTo0355706. Pain Physician 2008; 11:491-504.

71. Manchikanti L, Singh V, Falco FJ, Cash KA, Fellows B. Cervical medial branch blocks for chronic cervical facet joint pain: A randomized double-blind, controlled trial with one-year follow-up. Spine 2008; 33:1813-1820.

72. Manchikanti L, Singh V, Falco FJ, Cash KA, Pampati V. Lumbar facet joint nerve blocks in managing chronic facet joint pain: One-year follow-up of a randomized, double-blind controlled trial: Clinical Trial NCTo0355914. Pain Physician 2008; 11:121-132.

73. Manchikanti L, Cash KA, McManus CD, Pampati V, Smith HS. Preliminary results of randomized, equivalence trial of fluoroscopic caudal epidural injections in managing chronic low back pain: Part 1. Discogenic pain without disc herniation or radiculitis. Pain Physician 2008; 11:785-800.

74. Manchikanti L, Singh V, Cash KA, Pampati V, Damron KS, Boswell MV. Preliminary results of randomized, equivalence trial of fluoroscopic caudal epidural injections in managing chronic low back pain: Part 2. Disc herniation and radiculitis. Pain Physician 2008; 11:801-815.

75. Manchikanti L, Singh V, Cash KA, Pam pati V, Datta S. Preliminary results of randomized, equivalence trial of fluoroscopic caudal epidural injections in managing chronic low back pain: Part 3. Post surgery syndrome. Pain Physician 2008; 11:817-831.

76. Manchikanti L, Cash KA, McManus CD, Pampati V, Abdi S. Preliminary results of randomized, equivalence trial of flu- oroscopic caudal epidural injections in managing chronic low back pain: Part 4. Spinal stenosis. Pain Physician 2008; 11:833-848.

77. Berg AO, Allan JD. Introducing the third U.S. Preventive Services Task Force. Am J Prev Med 2001; 20:21-35.

78. Guyatt G, Gutterman D, Baumann MH, Addrizzo-Harris D, Hylek EM, Phillips B, Raskob G, Lewis SZ, Schünemann $H$. Grading strength of recommendations and quality of evidence in clinical guidelines. Report from an American College of Chest Physicians Task Force. Chest 2006; 129:174-181.

79. Freeman BJ, Fraser RD, Cain CM, Hall DJ, Chapple DC. A randomized, doubleblind, controlled trial: Intradiscal electrothermal therapy versus placebo for the treatment of chronic discogenic low back pain. Spine 2005; 30:2369-2377.

80. Pauza KJ, Howell S, Dreyfuss P. A randomized, placebo-controlled trial of intradiscal electrothermal therapy for the treatment of discogenic low back pain. Spine J 2004; 4:27-35.

81. Andersson GB, Mekhail NA, Block JE. Intradiscal electrothermal therapy (IDET). Spine 2006; 31:1402-1403.

82. Gibson JA, Waddell G. Letter to the editor. Spine 2006; 31:1402-1403.

83. Gerszten PC, Welch WC, McGrath PM, Willis SL. A prospective outcomes study of patients undergoing intradiscal electrothermy (IDET) for chronic low back pain. Pain Physician 2002; 5:360364.

84. Bryce DA, Nelson J, Glurich I, Berg RL. Intradiscal electrothermal annuloplasty therapy: A case series study leading to new considerations. WMJ 2005; 104:39-46.

85. Cohen SP, Larkin T, Abdi S, Chang A, Stojanovic M. Risk factors for failure and complications of intradiscal electrothermal therapy: A pilot study. Spine 2003; 28:1142-1147.

86. Derby R, Eek B, Lee SH, Seo KS, Kim BJ. Comparison of intradiscal restorative injections and intradiscal electrothermal treatment (IDET) in the treatment of low back pain. Pain Physician 2004; 7:63-66.

87. Derby R, Lee SH, Seo KS, Kazala K, Kim BJ, Kim MJ. Efficacy of IDET for relief of leg pain associated with discogenic low back pain. Pain Practice 2004; 4:281-285.

88. Freedman VA, Martin LG, Schoeni RF. Recent trends in disability and func- 
tioning among older adults in the United States. JAMA 2002; 288:3137-3146.

89. Saal JA, Saal JS. Intradiscal electrothermal treatment for chronic discogenic low back pain: Prospective outcome study with a minimum 2-year followup. Spine 2002; 279:966-973.

90. Karasek M, Bogduk N. Twelve-month follow-up of a controlled trial of intradiscal thermal anuloplasty for back pain due to internal disc disruption. Spine 2000; 25:2601-2607.

91. Lutz C, Lutz GE, Cooke PM. Treatment of chronic lumbar diskogenic pain with intradiscal electrothermal therapy: A prospective outcome study. Arch Phys Med Rehabil 2003; 84:23-28.

92. Lee MS, Cooper G, Lutz GE, Lutz C, Hong HM. Intradiscal Electrothermal Therapy (IDET) for treatment of chronic lumbar discogenic pain: A minimum 2year clinical outcome study. Pain Physician 2003; 6:443-448.

93. Webster BS, Verma S, Pransky GS. Outcomes of workers' compensation claimants with low back pain undergoing intradiscal electrothermal therapy. Spine 2004; 29:435-441.

94. Mekhail N, Kapural L. Intradiscal thermal annuloplasty for discogenic pain: An outcome study. Pain Practice 2004; 4:84-90.

95. Kapural L, Mekhail N, Korunda Z, Basali A. Intradiscal thermal annuloplasty for the treatment of lumbar discogenic pain in patients with multilevel degenerative disc disease. Anesth Analg 2004; 99:472-476.

96. Kapural L, Hayek S, Malak O, Arrigain S, Mekhail N. Intradiscal thermal annuloplasty versus intradiscal radiofrequency ablation for the treatment of discogenic pain: A prospective matched control trial. Pain Med 2005; 6:425-431.

97. Maurer P, Block JE, Squillante D. Intradiscal electrothermal therapy (IDET) provides effective symptom relief in patients with discogenic low back pain. J Spinal Disord Tech 2008; 21:55-62.

98. Nunley PD, Jawahar A, Brandao SM, Wilkinson K. Intradiscal electrothermal therapy (IDET) for low back pain in Worker's Compensation patients: Can it provide a potential answer? Longterm results. J Spinal Disord Tech 2008; 21:11-18.

99. Kapural L, Ng A, Dalton J, Mascha E, Kapural M, de la Garza M, Mekhail N. Intervertebral disc biacuplasty for the treatment of lumbar discogenic pain:
Results of a six-month follow-up. Pain Med 2008; 9:60-67.

100. Kapural L. Letter to the editor: Intervertebral disk cooled bipolar radiofrequency (intradiskal biacuplasty) for the treatment of lumbar diskogenic pain: 12 month follow up of the pilot study. Pain Med 2008; 9:407-408.

101. Ergun R, Sekerci Z, Bulut H, Dolgun H. Intradiscal electrothermal treatment for chronic discogenic low back pain: A prospective outcome study of $39 \mathrm{pa}$ tients with Oswestry Disability Index at 18 month follow up. Neurol Res 2008; 30:411-416.

102. Derby R, Eek B, Chen Y, O’Neill C, Ryan D. Intradiscal electrothermal annuloplasty (IDET): A novel approach for treating chronic discogenic back pain. Neuromodulation 2000; 3:82-88.

103. Saal JA, Saal JS. Intradiscal electrothermal treatment for chronic discogenic low back pain: A prospective outcome study with minimum 1-year follow-up. Spine 2000; 25:2622-2627.

104. Maurer P, Squillante D. Is intradiscal electrothermal annuloplasty (IDET) effective treatment for discogenic low back pain? A prospective cohort outcome study identifying successful patient selection criteria. Spine J 2002; 2: S37-S37.

105. Assietti R. Twenty-Four Months Outcome of 50 Patients Treated with Intradiscal Electrothermal Therapy (IDET) for Chronic Low Back Pain. Presented at the World Congress of Minimally Invasive Spine Surgery \& Techniques. Hawaii, 2008.

106. Endres SM, Fiedler GA, Larson KL. Effectiveness of intradiscal electrothermal therapy in increasing function and reducing chronic low back pain in selected patients. WMJ 2002; 101:31-34.

107. Welch WC, Gerszten PC, McGrath P. Intradiscal electrothermy: Indications, techniques, and clinical results. Clin Neurosurg 2001; 48:219-225.

108. Sluijter M, van Kleef M. Percutaneous intradiscal radio-frequency thermocoagulation. Spine 1996; 21:528-529.

109. Derby R, Seo KS, Kazala K, Chen YC, Lee $\mathrm{SH}$, Kim BJ. A factor analysis of lumbar intradiscal electrothermal annuloplasty outcomes. Spine J 2005; 5:256-261.

110. Singh V. Intradiscal electrothermal therapy: A preliminary report. Pain Physician 2000; 3:367-373.

111. Spruit M, Jacobs WC. Pain and function after intradiscal electrothermal treat- ment (IDET) for symptomatic lumbar disc degeneration. Eur Spine / 2002; 11:589-593.

112. Thompson K, Eckel T. Two-year results from the intradiscal electrothermal therapy nationwide registry. Spine J 2002; 2:S10.

113. Park SY, Moon SH, Park MS, Kim HS, Choi YJ, Lee HM. Intradiscal electrothermal treatment for chronic lower back pain patients with internal disc disruption. Yonsei Med J 2005; 46:539545.

114. Davis TT, Delamarter RB, Sra P, Goldstein TB. The IDET procedure for chronic discogenic low back pain. Spine 2004; 29:752-756.

115. Bogduk N, Karasek M. Two-year followup of a controlled trial of intradiscal electrothermal anuloplasty for chronic low back pain resulting from internal disc disruption. Spine / 2002; 2:343350.

116. Landsberger HA. Hawthorne Revisited: New York State School of Industrial and Labor Relations, Cornell University, Ithaca, New York, 1958.

117. Hrobjartsson A, Gotzsche PC. Is the placebo powerless? Update of a systematic review with 52 new randomized trials comparing placebo with no treatment. J Intern Med 2004; 256:91-100.

118. Hrobjartsson A, Gotzsche PC. Is the placebo powerless? An analysis of clinical trials comparing placebo with no treatment. N Engl J Med 2001; 344:15941602.

119. Rosenthal R, Jacobson L. Pygmalion in the Classroom: Teacher Expectation and Pupils' Intellectual Development. Irvington, New York, 1992.

120. Djurasovic M. Glassman D, Dimar JR 2nd, Johnson JR. Vertebral osteonecrosis associated with the use of intradiscal electrothermal therapy: A case report. Spine 2002; 27:E325-E328.

121. Schafer J, O'Connor D, Feinglass S, Salive M. Medicare evidence development and coverage advisory committee meeting on lumbar fusion surgery for treatment of chronic back pain from degenerative disc disease. Spine 2007; 32:2403-2404.

122. Krahn M, Naglie G. The next step in guideline development: Incorporating patient preferences. JAMA 2008; 300:436-438.

123. Montori V, Guyatt G. Progress in evidence-based medicine. JAMA 2008; 300:1814-1816. 